Geophysical and Astrophysical Fluid Dynamics

Vol. 00, No. 00, 00 Month 20xx, 1-23

\title{
On magnetostrophic inertia-less waves in quasi-geostrophic models of planetary cores
}

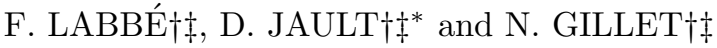 \\ †Univ. Grenoble Alpes, ISTerre, F-38000 Grenoble, France \\ $\ddagger$ CNRS, ISTerre, F-38000 Grenoble, France \\ (Received 00 Month 20xx; final version received 00 Month 20xx)
}

\begin{abstract}
We investigate quasi-geostrophic waves in a rotating spherical shell permeated by an imposed magnetic field $\overline{\boldsymbol{B}}$. A projection of the momentum equation onto a well chosen class of velocity fields results in a quasigeostrophic reduced model where, in contrast with previous such models, the evolution of the velocity is not prescribed by an equation for the axial vorticity. We consider fields $\overline{\boldsymbol{B}}$ that may be longitudinally dependent. Increasing the angular rotation frequency, we find that the non-axisymmetric Alfvén waves that are present at low rotation morph into inertial waves, torsional Alfvén waves and low frequency magnetostrophic waves that satisfy Taylor's constraint (i.e. vanishing acceleration of the geostrophic cylinders by the magnetic forces).
\end{abstract}

Keywords: Quasi-geostrophy; Magnetostrophic waves; Torsional waves; Taylor's constraint

\section{Introduction}

The development of models for Earth's core dynamics is guided by the characteristics of the waves that are present in the fluid outer core. These waves are influenced by both the fast rotation of the core and the magnetic field that permeates the fluid (Finlay 2007, Jault and Finlay 2015). Hide (1966) was the first to argue that deep two-dimensional hydromagnetic waves may be responsible for the geomagnetic secular variation (SV), with time scales of decades to centuries. He found two families of modes: high-frequency Rossby-type inertial modes and low-frequency magnetic modes that may be the source of the SV. Hide drew on the analogy between the equations for hydromagnetic waves in thick spherical shells of fluid and in thin spherical shells far from the equator. In the latter geometry, the (local) $\beta$-plane approximation gives a simple method to investigate small-amplitude planetary waves. Shortly after, remarking that there was limited evidence, at that time, for two-dimensionality in the observed SV, Malkus (1967) searched for three-dimensional modes in a full sphere. For a special choice of basic magnetic field, he found that both fast and slow hydromagnetic modes can be calculated from the knowledge of the solutions of the non-magnetic problem, that is, the inertial modes. Zhang et al. (2003) improved upon Malkus' work and obtained general explicit solutions from analytical expressions of the inertial waves (Zhang et al. 2001). Twodimensional hydromagnetic waves either fast or slow form a special class of solutions within this much wider ensemble.

Interest for these two-dimensional waves has nonetheless been rekindled by the numerical investigations of the geodynamo. Outside the cylindrical surface tangent to the inner core aligned with the rotation axis, the flow pattern of geodynamo simulations is often dominated by columnar convection, which is almost invariant along the rotation axis (see e.g. Christensen

*Corresponding author. Email: dominique.jault@ujf-grenoble.fr 
et al. 1999). This has been attributed to the small value of the Lehnert number, defined as the ratio of the period of the inertial waves to the period of the Alfvén waves (Jault 2008). This is also in agreement with the predominant equatorial symmetry of core surface flows estimated from modern satellite magnetic data (Gillet et al. 2011). Accordingly, Canet et al. (2014) have restricted their recent study of hydromagnetic waves to quasi-geostrophic (QG) solutions, which are almost invariant in the axial direction. Their model includes the spherical shell geometry rather than the local $\beta$-plane initially used by Hide (1966). Canet et al. (2014) have retrieved both the fast and the slow modes, with similar scaling of their frequencies as Hide (1966).

The slow magnetic modes are magnetostrophic inasmuch as viscosity is ignored in their calculation and inertia is subdominant: the Coriolis force is balanced by the pressure and Lorentz forces. Also, the kinetic energy of these modes is negligible in comparison with their magnetic energy. This is another key feature of the magnetostrophic regime. Taylor (1963) pioneered the investigation of this regime and stressed its importance in the context of the Earth's dynamo problem. He derived a condition on the Lorentz force, which today is referred to as Taylor's constraint: the circulation of the Lorentz force over the geostrophic surfaces (the fluid cylinders $\mathcal{C}(s)$ of radius $s$ and concentric with the rotation axis) averages to zero,

$$
\int_{\mathcal{C}(s)}((\nabla \times \boldsymbol{B}) \times \boldsymbol{B}) \cdot \mathbf{1}_{\phi} s \mathrm{~d} \phi \mathrm{d} z=0
$$

where $\left(\mathbf{1}_{s}, \mathbf{1}_{\phi}, \mathbf{1}_{z}\right)$ are the unit vectors in cylindrical coordinates $(s, \phi, z)$. There are very few examples of magnetic fields that satisfy Taylor's constraint (Livermore et al. 2009). Recently, $\mathrm{Wu}$ and Roberts (2015) have found an algorithm to calculate $\alpha^{2}$ and $\alpha \omega$ axisymmetric dynamos that are magnetostrophic. Jault (1995) had previously exhibited another instance of $\alpha \omega$ dynamo in a Taylor state. He scrutinized the transition to a Taylor state for a special example devised by Braginsky (1978) and found that it occurs close to the point at which two modes are kinematically unstable. When the basic magnetic field $\overline{\boldsymbol{B}}$ is symmetric about the rotation axis, the low frequency magnetic waves are longitudinally dependent and satisfy a linear version of Taylor's constraint automatically,

$$
\int_{\mathcal{C}(s)}((\boldsymbol{\nabla} \times \overline{\boldsymbol{B}}) \times \widetilde{\boldsymbol{B}}+(\boldsymbol{\nabla} \times \widetilde{\boldsymbol{B}}) \times \overline{\boldsymbol{B}}) \cdot \mathbf{1}_{\phi} s \mathrm{~d} \phi \mathrm{d} z=0
$$

where $\widetilde{\boldsymbol{B}}$ is the perturbation from the background state. In order to make the condition (1b) non-trivial, $\overline{\boldsymbol{B}}$ has to deviate from axisymmetry. Then, torsional (cylindrical) Alfvén waves may be part of the set of wave solutions as anticipated long ago by Gans (1971).

The torsional Alfvén waves consist of oscillations $u_{G}(s, t) \mathbf{1}_{\phi}$ of geostrophic cylinders. Their restoring mechanism is the magnetic torque on these cylinders induced by the shearing of $\bar{B}_{s}$ by $u_{G}$ (Braginsky 1970). Up to now, the torsional waves have always been investigated independently from the fast and slow hydromagnetic waves described above, though the latter waves are also strongly influenced by rotation. In this paper, we consider a non-zonal basic magnetic field $\bar{B}$ that enables us to examine simultaneously all these waves.

Using a variational approach, we set up the equations for quasi-geostrophic hydromagnetic waves in $\S 2$. We present our QG wave solution for the basic magnetic field of Malkus (1967), which is symmetric about the rotation axis, in $\S 3$. In the following section $\S 4$, we show the solution for a basic field that is not axisymmetric and presents one or two wavenumbers in addition to $m=0$. It includes torsional Alfvén waves and slow magnetostrophic waves. We end with concluding remarks in $\S 5$. 


\section{Reduced model with axially invariant magnetic and velocity fields}

\subsection{Exact MHD equations}

We model the Earth's fluid core as a spherical rotating shell of inner radius $r_{i}$ and outer radius $r_{o}$ permeated by a magnetic field $\overline{\boldsymbol{B}}$. The Navier-Stokes equation,

$$
\rho\left(\frac{\partial \boldsymbol{u}}{\partial t}+\boldsymbol{u} \cdot \nabla \boldsymbol{u}+2 \boldsymbol{\Omega} \times \boldsymbol{u}\right)=-\nabla \Pi+\frac{1}{\mu_{0}}(\boldsymbol{\nabla} \times \boldsymbol{B}) \times \boldsymbol{B}+\rho \nu \nabla^{2} \boldsymbol{u},
$$

gives the time evolution of the flow velocity $\boldsymbol{u}$, with $\boldsymbol{B}$ the magnetic field, $\Pi$ the reduced pressure, $\rho$ the fluid density, $\nu$ the kinematic viscosity and $\mu_{0}$ the magnetic permeability of free space. Terms on the left hand side of (2) describe the flow acceleration, where $2 \boldsymbol{\Omega} \times \boldsymbol{u}$ is the Coriolis acceleration in the rotating frame of angular velocity $\boldsymbol{\Omega}=\boldsymbol{\Omega} \mathbf{1}_{\mathbf{z}}$. Terms on the right hand side are, from left to right: the pressure gradient, the Lorentz force and the viscous force. The evolution of the magnetic field is governed by the induction equation,

$$
\frac{\partial \boldsymbol{B}}{\partial t}=\nabla \times(\boldsymbol{u} \times \boldsymbol{B})+\eta \nabla^{2} \boldsymbol{B}
$$

where $\eta$ is the magnetic diffusivity. Both $\boldsymbol{u}$ and $\boldsymbol{B}$ are divergence-less:

$$
\boldsymbol{\nabla} \cdot \boldsymbol{u}=0, \quad \boldsymbol{\nabla} \cdot \boldsymbol{B}=0
$$

We non-dimensionalize the equations using $r_{0}$ as the unit length and $B_{0}$ (a measure of the intensity of $\overline{\boldsymbol{B}}$ ) as the magnetic field strength. The velocity field is scaled by the Alfvén wave velocity $V_{A}=\frac{B_{0}}{\left(\rho \mu_{0}\right)^{1 / 2}}$, which yields the unit time $T_{A}=r_{0} / V_{A}$ and the pressure scale $\rho V_{A}^{2}$. The non-dimensional Navier-Stokes and induction equations are then

$$
\begin{gathered}
\frac{\partial \boldsymbol{u}}{\partial t}+\boldsymbol{u} \cdot \nabla \boldsymbol{u}+\frac{2}{\mathrm{Le}} \mathbf{1}_{\boldsymbol{z}} \times \boldsymbol{u}=-\nabla \Pi+(\boldsymbol{\nabla} \times \boldsymbol{B}) \times \boldsymbol{B}+\frac{\mathrm{P}_{\mathrm{m}}}{\mathrm{Lu}} \nabla^{2} \boldsymbol{u}, \\
\frac{\partial \boldsymbol{B}}{\partial t}=\boldsymbol{\nabla} \times(\boldsymbol{u} \times \boldsymbol{B})+\mathrm{Lu}^{-1} \nabla^{2} \boldsymbol{B} .
\end{gathered}
$$

The three dimensionless numbers of our system can be introduced as the ratios of time scales. The Lehnert number,

$$
\mathrm{Le} \equiv \frac{T_{\Omega}}{T_{A}}=\frac{B_{0}}{\Omega r_{0}\left(\rho \mu_{0}\right)^{1 / 2}},
$$

measures the Alfvén waves time-scale $T_{A}$ against the rotation time-scale $T_{\Omega}=\Omega^{-1}$. The Lundquist number,

$$
\mathrm{Lu} \equiv \frac{T_{\eta}}{T_{A}}=\frac{r_{0} B_{0}}{\eta\left(\rho \mu_{0}\right)^{1 / 2}}
$$

compares the magnetic diffusion time scale $T_{\eta}=r_{0}^{2} / \eta$ and $T_{A}$. The magnetic Prandtl number,

$$
\mathrm{P}_{\mathrm{m}} \equiv \frac{T_{\eta}}{T_{\nu}}=\frac{\nu}{\eta},
$$

is the ratio of the magnetic and viscous dissipation times.

For the Earth's core, Le is estimated to be about $10^{-4}$ (Gillet et al. 2010), Lu is of the order of $10^{5}$ and $\mathrm{P}_{\mathrm{m}} \sim 10^{-6}$ (Pozzo et al. 2013). On account of the large value of Lu, we shall thus neglect the dissipation terms. Furthermore, the amplitude of the fluid flow $U$ is much weaker than $V_{A}$ in geophysical applications, as measured by the Alfvén number $\mathrm{Al} \equiv U / V_{A} \sim 10^{-2}$. This translates to a magnetic energy about $10^{4}$ times larger than the kinetic energy. For this 
reason, we include only a dynamo magnetic field - and not a velocity field - in the background state. Then, in linearized form $(\boldsymbol{B}=\overline{\boldsymbol{B}}+\widetilde{\boldsymbol{B}})$, equations (5) and (6) become:

$$
\begin{gathered}
\frac{\partial \boldsymbol{u}}{\partial t}+\frac{2}{\mathrm{Le}} \mathbf{1}_{\boldsymbol{z}} \times \boldsymbol{u}=-\boldsymbol{\nabla} \Pi+(\boldsymbol{\nabla} \times \overline{\boldsymbol{B}}) \times \widetilde{\boldsymbol{B}}+(\boldsymbol{\nabla} \times \widetilde{\boldsymbol{B}}) \times \overline{\boldsymbol{B}} \\
\frac{\partial \widetilde{\boldsymbol{B}}}{\partial t}=\boldsymbol{\nabla} \times(\boldsymbol{u} \times \overline{\boldsymbol{B}}) .
\end{gathered}
$$

We note that geodynamo simulations commonly present values of $\mathrm{Al}$ about unity (e.g., Soderlund et al. 2012). As a consequence such models may not be relevant to understand the geomagnetic SV at periods from $T_{A}$ to $T_{U}=r_{o} / U$, i.e. from interannual to centennial timescales. This constitutes another motivation for studies relying on imposed fields. Along the same lines, Teed et al. (2015) recently investigated torsional waves in magnetoconvection simulations (i.e. by imposing the field at the outer spherical surface). By exploring solutions for small values of the Ekman number $\left(E=T_{\Omega} / T_{\nu}<10^{-6}\right)$ that are difficult to achieve in present dynamo simulations, Teed et al. duly found torsional waves driven by Lorentz forces (rather than by Reynolds forces as in their dynamo runs).

\subsection{Quasi-geostrophic approximation}

Geostrophy describes the state of a rapidly rotating fluid, in which the pressure gradient equilibrates the action of the Coriolis force. Any geostrophic flow $\boldsymbol{u}$ therefore satisfies the geostrophic balance:

$$
\frac{2}{\operatorname{Le}}\left(\mathbf{1}_{\boldsymbol{z}} \times \boldsymbol{u}\right)=-\boldsymbol{\nabla} \Pi
$$

Projecting (10) onto $\mathbf{1}_{\boldsymbol{z}}$ yields the Proudman-Taylor theorem, which indicates invariance of the flow along the rotation axis. It is therefore convenient to work with cylindrical coordinates. The shape of the container is then defined by $H(s)$, the half-height of geostrophic columns. For a sphere of radius unity, $H(s)=\left(1-s^{2}\right)^{1 / 2}$. This holds also in a spherical shell for $s>s_{i}$, where $s_{i}$ is the radius of the inner boundary (see Figure 1). In a container symmetric about the rotation axis and with variable height, pure geostrophic motions consist in rigid rotations of cylindrical shells $u_{G}(s) \mathbf{1}_{\phi}$.

The time evolution of the geostrophic (and zonal) flow $u_{G}(s, t)$ is directly governed by the $\phi$-component of the momentum equation integrated over the geostrophic cylinders $\mathcal{C}(s)$,

$$
\forall s, \quad \frac{\partial \zeta_{G}}{\partial t}=\frac{1}{4 \pi s H} \oint\{(\boldsymbol{\nabla} \times \overline{\boldsymbol{B}}) \times \widetilde{\boldsymbol{B}}+(\boldsymbol{\nabla} \times \widetilde{\boldsymbol{B}}) \times \overline{\boldsymbol{B}}\} \cdot \mathbf{1}_{\phi} \mathrm{d} \phi,
$$

with

$$
\{X\}=\int_{-H}^{H} X \mathrm{~d} z
$$

and the geostrophic angular velocity defined by

$$
\zeta_{G} \equiv \frac{u_{G}}{s} .
$$

In this paper, we make the assumption that the non-zonal velocity is nearly geostrophic. We take advantage of the relatively weak $z$-dependence induced by the rapid rotation (Gillet and Jones 2006) and we assume a priori $z$-invariance of the velocity $\boldsymbol{u}_{\perp}$ perpendicular to the rotation axis (Canet et al. 2014, Jault and Finlay 2015). From the solenoidal condition (4a) for the flow and the no-penetration boundary condition,

$$
u_{z}= \pm \boldsymbol{u}_{\perp} \cdot \nabla H \quad \text { at } \quad z= \pm H
$$




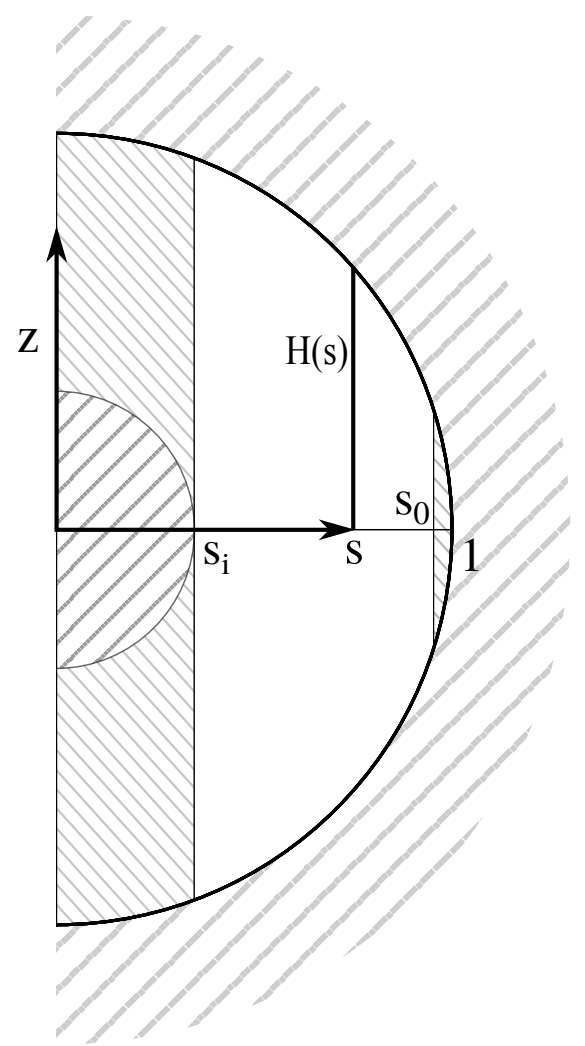

Figure 1. Schematics of the model (meridional cross-section), which excludes a region close to $s=1$.

we infer that $u_{z}$ varies linearly with $z$ and that $\boldsymbol{\nabla} \cdot\left(H \boldsymbol{u}_{\perp}\right)=0$. Hence, there is a stream function $\psi(s, \phi, t)$ such that

$$
\boldsymbol{u}_{\perp}(\psi)=\frac{1}{H} \boldsymbol{\nabla} \times\left(\psi \mathbf{1}_{\boldsymbol{z}}\right),
$$

and the total flow is also function of $\psi$ :

$$
\boldsymbol{u}(\psi)=\frac{1}{H} \boldsymbol{\nabla} \times\left(\psi \mathbf{1}_{\boldsymbol{z}}\right)+z \frac{\beta}{s H} \frac{\partial \psi}{\partial \phi} \mathbf{1}_{\boldsymbol{z}} \quad \text { with } \quad \beta=\frac{1}{H} \frac{\mathrm{d} H}{\mathrm{~d} s} .
$$

Note that the expression of Schaeffer and Cardin (2005) as a function of $\Psi=\psi / H$ is equivalent. The geostrophic part $\psi_{G}$ of $\psi$ is treated separately:

$$
\psi_{G}(s, t)=\frac{1}{2 \pi} \oint \psi(s, \phi, t) \mathrm{d} \phi, \quad \zeta_{G}(s, t)=-\frac{1}{s H} \frac{\partial \psi_{G}}{\partial s}
$$

(see the equation for $\zeta_{G}$ above). Finally, investigating $\psi$ in the domain $s_{i} \leq s \leq 1$, we need boundary conditions for its non-zonal part $\psi^{N Z}=\psi-\psi_{G}$ at $s=s_{i}$ and $s=1$. Regularity at $s=1$ imposes

$$
\forall t, \quad \psi^{N Z}(1)=0
$$

Similarly, we set

$$
\forall t, \quad \psi^{N Z}\left(s_{i}\right)=0,
$$

so that no fluid is allowed to leave the volume. For the geostrophic angular velocity, we have

$$
\forall t, \quad \zeta_{G}\left(s_{i}\right)=\zeta_{G}(1)=0 .
$$


The velocity fields $\boldsymbol{u}$ that are solutions of the momentum equation (8) are generally not exactly of the form (12c). As a result, we cannot simply substitute (12c) for $\boldsymbol{u}$ in (8) to get the evolution equation of $\psi$. We seek instead a reduced model that approximates (8) and admits solutions of the form $(12 \mathrm{c})$. In this paper, we follow a variational approach and obtain the equation that governs the time evolution of $\psi$ by projecting (8) onto trial functions $\boldsymbol{u}^{\prime}\left(\psi^{\prime}\right)$ of the form (12c). Taken together, the conditions (4a), (12a), and (12g,h) ensure that the pressure term is eliminated through this operation:

$$
-\iiint_{\mathcal{V}} \boldsymbol{u}^{\prime} \cdot \nabla \Pi \mathrm{d} \mathcal{V}=0, \quad \text { with } \quad \mathrm{d} \mathcal{V}=s \mathrm{~d} s \mathrm{~d} \phi \mathrm{d} z
$$

Integrating over $z$, then integrating by parts over $s$ and $\phi$, the contribution of the Coriolis term gives:

$$
\begin{aligned}
\iiint_{\mathcal{V}} \boldsymbol{u}^{\prime} \cdot\left(\frac{2}{\mathrm{Le}} \mathbf{1}_{\boldsymbol{z}} \times \boldsymbol{u}\right) \mathrm{d} \mathcal{V} & =\frac{4}{\mathrm{Le}} \iint_{\mathcal{A}}\left(\frac{1}{H} \frac{\partial \psi^{\prime}}{\partial \phi} \frac{\partial \psi}{\partial s}-\frac{1}{H} \frac{\partial \psi^{\prime}}{\partial s} \frac{\partial \psi}{\partial \phi}\right) \mathrm{d} \phi \mathrm{d} s \\
& =\frac{4}{\operatorname{Le}} \iint_{\mathcal{A}} \psi^{\prime} \frac{\partial \psi}{\partial \phi} \frac{\mathrm{d}}{\mathrm{d} s}\left(\frac{1}{H}\right) \mathrm{d} \phi \mathrm{d} s \\
& =-\frac{4}{\mathrm{Le}} \iint_{\mathcal{A}} \psi^{\prime} \frac{\beta}{s H} \frac{\partial \psi}{\partial \phi} \mathrm{d} \mathcal{A},
\end{aligned}
$$

with $\mathcal{A}$ the annulus $s_{i} \leq s \leq 1$ of surface element $\mathrm{d} \mathcal{A}=s \mathrm{~d} s \mathrm{~d} \phi$. We note that

$$
\begin{aligned}
\iiint_{\mathcal{V}} \boldsymbol{u}\left(\psi_{1}\right) \cdot \boldsymbol{u}\left(\psi_{2}\right) \mathrm{d} \mathcal{V} & =-2 \iint_{\mathcal{A}} \psi_{1}\left(\frac{1}{s^{2} H} \frac{\partial^{2} \psi_{2}}{\partial \phi^{2}}+\frac{1}{s} \frac{\partial}{\partial s}\left(\frac{s}{H} \frac{\partial \psi_{2}}{\partial s}\right)+\frac{H \beta^{2}}{3 s^{2}} \frac{\partial^{2} \psi_{2}}{\partial \phi^{2}}\right) \mathrm{d} \mathcal{A} \\
& =-2 \iint_{\mathcal{A}} \psi_{1} \mathcal{L}^{2} \psi_{2} \mathrm{~d} \mathcal{A}
\end{aligned}
$$

where

$$
\mathcal{L}^{2} \psi=\nabla \cdot\left(\frac{1}{H} \nabla \psi\right)+\frac{H \beta^{2}}{3 s^{2}} \frac{\partial^{2} \psi}{\partial \phi^{2}} .
$$

As a result, the acceleration term yields

$$
\iiint_{\mathcal{V}} \boldsymbol{u}^{\prime} \cdot \frac{\partial \boldsymbol{u}}{\partial t} \mathrm{~d} \mathcal{V}=-2 \iint_{\mathcal{A}} \psi^{\prime} \frac{\partial}{\partial t}\left(\mathcal{L}^{2} \psi\right) \mathrm{d} \mathcal{A}
$$

Leaving aside for a while the magnetic forces, we obtain by (13b) (where in the absence of magnetic field, we have redimensionalized time by the rotation time-scale $\Omega^{-1}$ ) and (13e) the equation for Rossby waves,

$$
\frac{\partial}{\partial t} \mathcal{L}^{2} \psi+\frac{2 \beta}{s H} \frac{\partial \psi}{\partial \phi}=0 .
$$

The notation $\beta$ for the slope parameter emphasizes the analogy with the vorticity equation in the $\beta$-plane model (Vallis 2006, p. 176). However, equation (13f) should not be mistaken for the axial vorticity equation, which was an essential feature of previous quasi-geostrophic models in a rapidly rotating sphere (Gillet and Jones 2006, Calkins et al. 2012, Canet et al. 2014). The two equations can be formulated similarly, but with different definitions for $\mathcal{L}^{2}$. The operator $\mathcal{L}_{\zeta}^{2}$ that enters the $z$-component of the vorticity equation is

$$
\mathcal{L}_{\zeta}^{2} \psi=\nabla \cdot\left(\frac{1}{H} \nabla \psi\right)
$$

rather than (13d). 
There now remains to calculate

$$
\iiint_{\mathcal{V}} \boldsymbol{u}^{\prime} \cdot((\boldsymbol{\nabla} \times \overline{\boldsymbol{B}}) \times \widetilde{\boldsymbol{B}}+(\boldsymbol{\nabla} \times \widetilde{\boldsymbol{B}}) \times \overline{\boldsymbol{B}}) \mathrm{d} \mathcal{V} .
$$

\subsection{Magnetic potential}

By writing a relation linking $\boldsymbol{B}$ to a magnetic potential $A$ similar to the equation (12) defining $\boldsymbol{u}$ as a function of the stream function $\psi$, one can enforce the divergence-free property of the magnetic field in the same way as the mass conservation [see Tobias et al., 2007 in the $\beta$-plane context or Zeitlin, 2013 in the framework of rotating shallow water magnetohydrodynamics]. We will assume:

$$
\overline{\boldsymbol{B}}=\frac{1}{H} \boldsymbol{\nabla} \times\left(\bar{A} \mathbf{1}_{\boldsymbol{z}}\right)+z \frac{\beta}{s H} \frac{\partial \bar{A}}{\partial \phi} \mathbf{1}_{\boldsymbol{z}} .
$$

Our definition implies that the field lines of $\overline{\boldsymbol{B}}$ close in the fluid. From a geophysical point of view, this property can be justified by the radial field $\bar{B}_{r}$ being small on the core surface compared to its value inside the core (see Gillet et al. 2010). We choose also the same boundary conditions for the magnetic potential as for $\psi$ :

$$
\frac{\partial \bar{A}}{\partial \phi}\left(s_{i}\right)=\frac{\partial \bar{A}}{\partial \phi}(1)=0
$$

If $\boldsymbol{u}$ is of the form (12) and $\overline{\boldsymbol{B}}$ of the form (16), then $\widetilde{\boldsymbol{B}}$ is also of this type:

$$
\widetilde{\boldsymbol{B}}=\frac{1}{H} \boldsymbol{\nabla} \times\left(\widetilde{A} \mathbf{1}_{\boldsymbol{z}}\right)+z \frac{\beta}{s H} \frac{\partial \widetilde{A}}{\partial \phi} \mathbf{1}_{\boldsymbol{z}}
$$

(and the magnetic potential $\widetilde{A}$ satisfies the same boundary condition as $\bar{A}$ ).

We have indeed

$$
\boldsymbol{\nabla} \times(\boldsymbol{u} \times \boldsymbol{B})=\frac{1}{H} \boldsymbol{\nabla} \times\left(C \mathbf{1}_{\boldsymbol{z}}\right)+z \frac{\beta}{s H} \frac{\partial C}{\partial \phi} \mathbf{1}_{\boldsymbol{z}}
$$

with

$$
C=\frac{1}{H} \mathcal{J}(\psi, A),
$$

and $\mathcal{J}$, the Jacobian operator:

$$
\mathcal{J}(X, Y)=\frac{1}{s}\left(\frac{\partial X}{\partial s} \frac{\partial Y}{\partial \phi}-\frac{\partial Y}{\partial s} \frac{\partial X}{\partial \phi}\right) .
$$

The induction equation thus becomes:

$$
\frac{\partial \widetilde{A}}{\partial t}=\frac{1}{H} \mathcal{J}(\psi, \bar{A}) .
$$

Separating the zonal and non-zonal flows in the induction equation gives

$$
\frac{\partial \widetilde{A}}{\partial t}=-\zeta_{G} \frac{\partial \bar{A}}{\partial \phi}+\frac{1}{H} \mathcal{J}\left(\psi^{N Z}, \bar{A}\right) .
$$

We calculate now the projection of the magnetic force on the trial functions $\boldsymbol{u}^{\prime}$ introduced in $\S 2.2$,

$$
\begin{aligned}
& \iiint_{\mathcal{V}} \boldsymbol{u}^{\prime}\left(\psi^{\prime}\right) \cdot((\boldsymbol{\nabla} \times \boldsymbol{B}) \times \boldsymbol{B}) \mathrm{d} \mathcal{V}=-\iiint_{\mathcal{V}}(\boldsymbol{\nabla} \times \boldsymbol{B}) \cdot\left(\boldsymbol{u}^{\prime}\left(\psi^{\prime}\right) \times \boldsymbol{B}\right) \mathrm{d} \mathcal{V} \\
& =-\iiint_{\mathcal{V}} \boldsymbol{B} \cdot \boldsymbol{\nabla} \times\left(\boldsymbol{u}^{\prime}\left(\psi^{\prime}\right) \times \boldsymbol{B}\right) \mathrm{d} \mathcal{V}-\iiint_{\mathcal{V}} \boldsymbol{\nabla} \cdot\left(\boldsymbol{B} \times\left(\boldsymbol{u}^{\prime}\left(\psi^{\prime}\right) \times \boldsymbol{B}\right)\right) \mathrm{d} \mathcal{V} .
\end{aligned}
$$


Both $\boldsymbol{u}^{\prime}$ and $\boldsymbol{B}$ have zero normal components at the boundaries so that the last integral is null. The remaining integral on the right hand side is of the form

$$
-\iiint_{\mathcal{V}} \boldsymbol{B}(A) \cdot \boldsymbol{v}\left(\psi_{2}\right) \mathrm{d} \mathcal{V} \quad \text { with } \quad \psi_{2}=\frac{1}{H} \mathcal{J}\left(\psi^{\prime}, A\right)
$$

and $\boldsymbol{v}$ of the form (12) (see the relation (18a)). Using (13c), (19a) becomes

$$
\begin{aligned}
& \iiint_{\mathcal{V}} \boldsymbol{u}^{\prime}\left(\psi^{\prime}\right) \cdot((\boldsymbol{\nabla} \times \boldsymbol{B}) \times \boldsymbol{B}) \mathrm{d} \mathcal{V}=-2 \iint_{\mathcal{A}} \psi_{2} \mathcal{L}^{2} A \mathrm{~d} \mathcal{A} \\
& =2 \iint_{\mathcal{A}} \psi^{\prime} \mathcal{J}\left(A, \frac{1}{H} \mathcal{L}^{2} A\right) \mathrm{d} \mathcal{A} .
\end{aligned}
$$

Equations (13) and (19c) imply

$$
\left[\frac{\partial}{\partial t} \mathcal{L}^{2}+\frac{2}{\operatorname{Le}} \frac{\beta}{s H} \frac{\partial}{\partial \phi}\right] \psi^{N Z}=-\left(\mathcal{J}\left(\bar{A}, \frac{1}{H} \mathcal{L}^{2} \widetilde{A}\right)+\mathcal{J}\left(\widetilde{A}, \frac{1}{H} \mathcal{L}^{2} \bar{A}\right)\right)^{N Z},
$$

which gives the time evolution of the non-zonal part $\psi^{N Z}$ of $\psi$.

The equation (11) for the geostrophic velocity can be transformed into

$$
\frac{\partial \zeta_{G}}{\partial t}=-\frac{1}{2 \pi s^{3} H} \oint\left(\frac{\partial}{\partial s}\left(\frac{s}{H} \frac{\partial \bar{A}}{\partial s}\right) \frac{\partial \widetilde{A}}{\partial \phi}+\frac{\partial \bar{A}}{\partial \phi} \frac{\partial}{\partial s}\left(\frac{s}{H} \frac{\partial \widetilde{A}}{\partial s}\right)\right) \mathrm{d} \phi .
$$

In Appendix A, we show that it is possible to generalize the reduced equations (18e), (19d) and (20) by including both the non-linear and the dissipative terms.

\section{4. $\quad$ Numerical method}

We investigate the linear system $(18 \mathrm{e}, 19 \mathrm{~d}, 20)$ as an eigenvalue problem. We look for solutions of the form

$$
[\psi, \widetilde{A}](s, \phi, t)=\sum_{m}\left[\psi_{m}^{\omega}(s), \widetilde{A_{m}^{\omega}}(s)\right] \exp [-\mathrm{i}(m \phi+\omega t)]
$$

where $m$ is an integer that can be of either sign. The series for $\widetilde{A}$ are truncated at $|m|=10$, as adding further terms does not change significantly the small $m(m \leq 6)$ solution when the basic field is itself large-scale. We rely on finite differences methods to represent the radial dependence of $\psi_{m}$ and $\widetilde{A}_{m}$ and use centered derivatives with second order precision. We have used up to 200 uniformly distributed radial grid points, although most of our calculations have been done using only 50 points, as computation time increases drastically with the size of the problem. In order to search for solutions to the eigenvalue problem, we use LAPACK $^{\circledR}$ routines available from the Matlab ${ }^{\circledR}$ software. For each eigenfrequency $\omega$, there is a counterpart $-\omega$ of opposite sign. Examining the eigenvectors associated to these frequencies, we note that $\psi_{-m}^{-\omega}(s)$ and $\widetilde{A_{-m}^{-\omega}}(s)$ are complex conjugates of $\psi_{m}^{\omega}(s)$ and $\widetilde{A_{m}^{\omega}}(s)$. Adding the solutions for $+\omega$ and $-\omega$, we find that the real solutions can be written as

$$
\begin{aligned}
\widetilde{A}(s, \phi, t)= & \operatorname{Re}\left(\widetilde{A_{0}^{\omega}}\right) \cos (\omega t)+\operatorname{Im}\left(\widetilde{A_{0}^{\omega}}\right) \sin (\omega t) \\
& +\sum_{m>0}\left[\operatorname{Re}\left(\widetilde{A_{-m}^{\omega}}\right) \cos (m \phi-\omega t)-\operatorname{Im}\left(\widetilde{A_{-m}^{\omega}}\right) \sin (m \phi-\omega t)\right] \\
& +\sum_{m>0}\left[\operatorname{Re}\left(\widetilde{A_{m}^{\omega}}\right) \cos (m \phi+\omega t)+\operatorname{Im}\left(\widetilde{A_{m}^{\omega}}\right) \sin (m \phi+\omega t)\right]
\end{aligned}
$$


with an analogous expression for $\psi(s, \phi)$ except for the axisymmetric part of $\psi$ which is described separately as a function of $\zeta_{G}(s)$. The first line of equation (21b) describes a zonal wave oscillating with frequency $\omega$, the second one describes a prograde wave, and the third one a retrograde wave.

\section{Malkus' basic state}

In this section, we assume symmetry about the rotation axis of the imposed field

$$
\overline{\boldsymbol{B}}=\bar{B}_{\phi}(s) \mathbf{1}_{\phi} .
$$

As a result, equations for the different wavenumbers $m$ separate, and no zonal flow is created.

\subsection{Theoretical background}

Malkus (1967) investigated three-dimensional hydromagnetic waves in a rotating fluid sphere. He remarked that assuming $\bar{B}_{\phi}(s)=s$, which corresponds to the field generated by a uniform current parallel to rotation axis, introduces significant simplifications (see also Zhang et al. (2003)). Equation (9) requires the velocity and magnetic field perturbations to be proportional. Furthermore, equations (8) and (9) can be combined into a new one, similar to the equation for purely hydrodynamic inertial waves. Malkus found that there are two sets of eigenfrequencies of the hydromagnetic system, which are related to the frequencies $\lambda$ of inertial waves through

$$
\omega^{ \pm}=\frac{1}{2 \operatorname{Le}} \lambda\left(1 \pm\left(1+\frac{4 \operatorname{Le}^{2} m(m-\lambda)}{\lambda^{2}}\right)^{1 / 2}\right) .
$$

Note that here the dimensional scale of the inertial frequencies $\lambda$ is $\Omega$ rather than the Alfvén frequency, which cannot be defined in the absence of magnetic field. Fast waves of frequency $\omega^{I}$ (denoted $\omega^{+}$in $(23)$ ) are dominated by inertial force, while slow waves of frequency $\omega^{M C}$ $\left(\omega^{-}\right)$are governed by Coriolis and magnetic forces, hence their name of $M C$ (Magneto-Coriolis) waves. In the limit of vanishing rotation $(\mathrm{Le} \rightarrow \infty),(23)$ transforms into the dispersion relation of Alfvén waves, $\omega^{ \pm}= \pm m$.

We are interested in the hydromagnetic waves that are related to a special class of hydrodynamic inertial waves, where the wave motions are nearly independent of the axis of rotation (Zhang et al. 2001). These slow quasi-geostrophic inertial waves (also known as Rossby waves) arise in a rotating fluid if the container has variable height. Zhang et al. (2001) derived a threedimensional (e.g. without the prior assumption of axial invariance) analytic solution for these Rossby waves. They found that their frequency, once scaled by $\Omega$, is accurately estimated as

$$
\lambda_{m, N} \sim-\frac{2}{m+2}\left[\left(1+\frac{m(m+2)}{N(2 N+2 m+1)}\right)^{1 / 2}-1\right],
$$

where $N$ has integer values $(N \geq 1)$ and increases with the complexity in the $s$-direction. The sign of the frequency, in (24), indicates that the Rossby waves always travel in the prograde direction (i.e. eastward in a geophysical context). Inserting $\lambda$ from (24) in (23), we readily obtain approximate expressions for the eigenfrequencies of hydromagnetic quasi-geostrophic waves. We verify that quasi-geostrophic $M C$ waves propagate in the retrograde (westward) direction as predicted by Hide (1966).

An alternative approach is to suppose from the beginning that the waves are twodimensional, as in $\S 2.3$. The expression (23) remains valid in the framework of the reduced QG model. With Malkus field $\left(\partial \bar{A} / \partial s=-s H, \mathcal{L}^{2} \bar{A}=-2 H\right)$, simplifications occur and $(18 \mathrm{~d}, 19 \mathrm{~d})$ 
can be written as:

$$
\begin{aligned}
{\left[\frac{\partial}{\partial t} \mathcal{L}^{2}+\frac{2 \beta}{\operatorname{Le} s H} \frac{\partial}{\partial \phi}\right] \psi } & =\left[\frac{\partial}{\partial \phi} \mathcal{L}^{2}+\frac{2 \beta}{s H} \frac{\partial}{\partial \phi}\right] \widetilde{A}, \\
\frac{\partial \widetilde{A}}{\partial t} & =\frac{\partial \psi}{\partial \phi} .
\end{aligned}
$$

Using the decomposition (21a), one can transform the set of equations (25) into

$$
\left(\omega^{2}-m^{2}\right) \mathcal{L}^{2} \psi+\frac{2 \beta}{\operatorname{Le} s H}\left(m \omega-m^{2} \mathrm{Le}\right) \psi=0,
$$

while the dispersion equation for Rossby waves (using $\Omega$ as frequency unit) is simply

$$
\lambda \mathcal{L}^{2} \psi+\frac{2 \beta m}{s H} \psi=0 .
$$

Finally, (26) can be transformed into (27) provided that $\omega$ satisfies (23).

Canet et al. (2014) studied numerically the two classes of waves (see their Figure 3), making the ansatz

$$
\boldsymbol{B}=\boldsymbol{\nabla} \times\left(A \mathbf{1}_{\boldsymbol{z}}\right)
$$

rather than (17). Then taking into account the axial velocity component, they cannot write the induction term as the curl of an axial vector. They made instead the small slope approximation and discarded the contribution of the axial velocity in the induction equation. In this small slope limit, their choice (14) for $\mathcal{L}^{2}$ (as they opt to use the $z$-component of the vorticity equation) does not differ much from (13d).

\subsection{Benchmarking}

We investigate the eigenmodes of our QG system in a full sphere and compare the results to the prediction from (23) for moderate values of $m, m \leq 30$ (figure 2). As Le is lowered, Alfvén waves turn into slow and fast waves. The two branches of hydromagnetic waves split when Le $\ll 1$. The eigenvalues of the system $(25)$ (i.e., system $(18 \mathrm{e}, 19 \mathrm{~d}, 20)$ simplified by the use of the Malkus field) compare remarkably well with the analytical solutions, except for the modes with the largest radial scale $(N<3)$. The model based on equation $(13 \mathrm{~d})$ clearly improves on the model using (14), in this configuration at least. Also, all $M C$ modes are retrograde.

Dimensionless magnetic and kinetic energies are:

$$
E_{B}=\frac{1}{2} \int_{\mathcal{V}} \widetilde{B}^{2} \mathrm{~d} \mathcal{V}, \quad E_{u}=\frac{1}{2} \int_{\mathcal{V}} \boldsymbol{u}^{2} \mathrm{~d} \mathcal{V}
$$

Figure 3 displays the ratio of magnetic energy to kinetic energy. It confirms that fast waves have an inertial nature, while slow waves are magnetostrophic: magnetic energy predominates.

\section{Taylor's constraint}

We add a non-axisymmetric component to the main field, by taking $\bar{A}$ of the form

$$
\bar{A}(s, \phi)=\bar{A}^{Z}(s)+\alpha f(s) \sum_{m>0} \frac{\cos (m \phi)}{m},
$$

where the superscript $Z$ denotes the zonal component. The parameter $\alpha$ will be varied between 0 and $10^{-1}$. By choosing $\bar{A}^{Z}(s)$ identical to $\bar{A}(s)$ from the previous section, setting the 

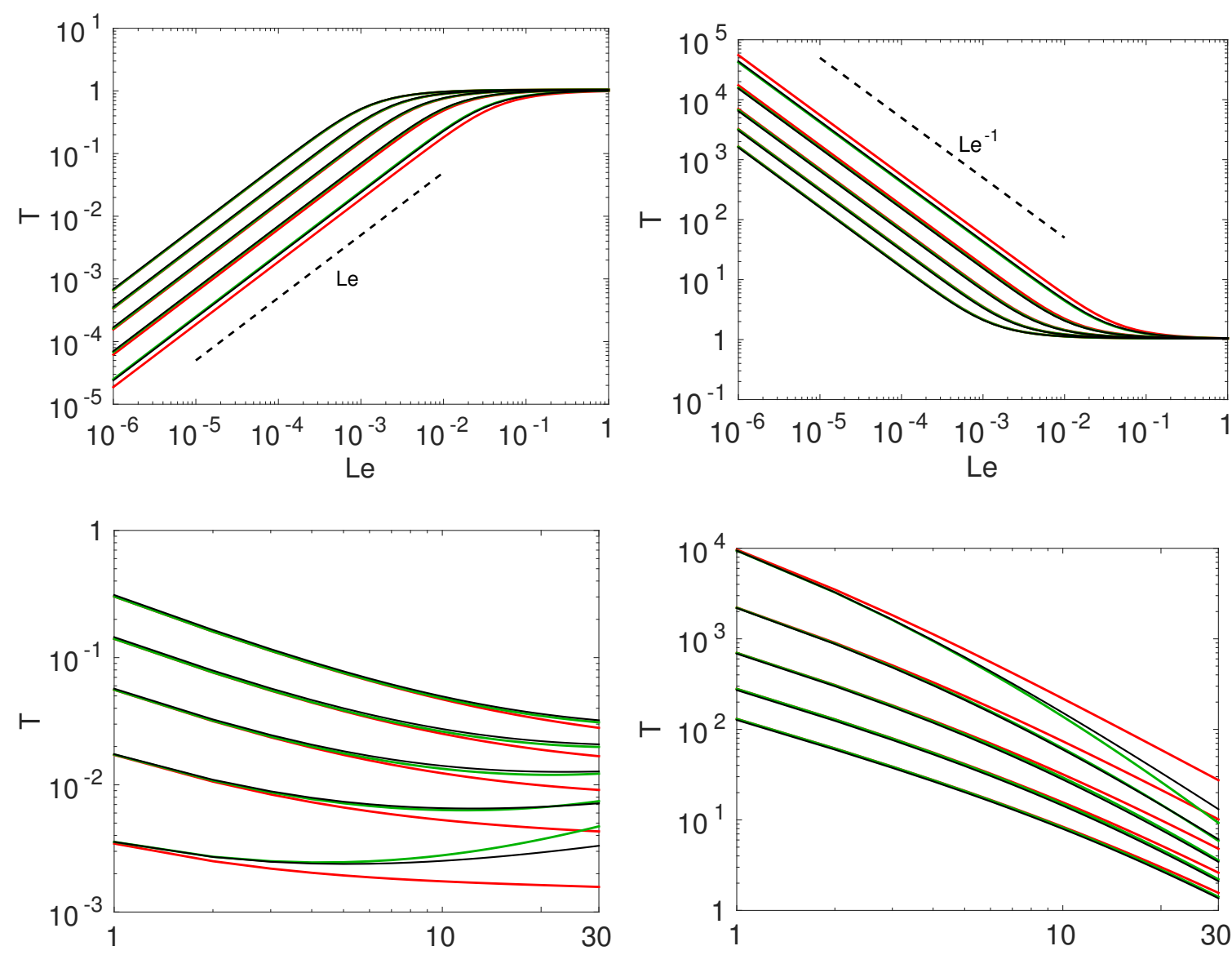

m

$\mathrm{m}$
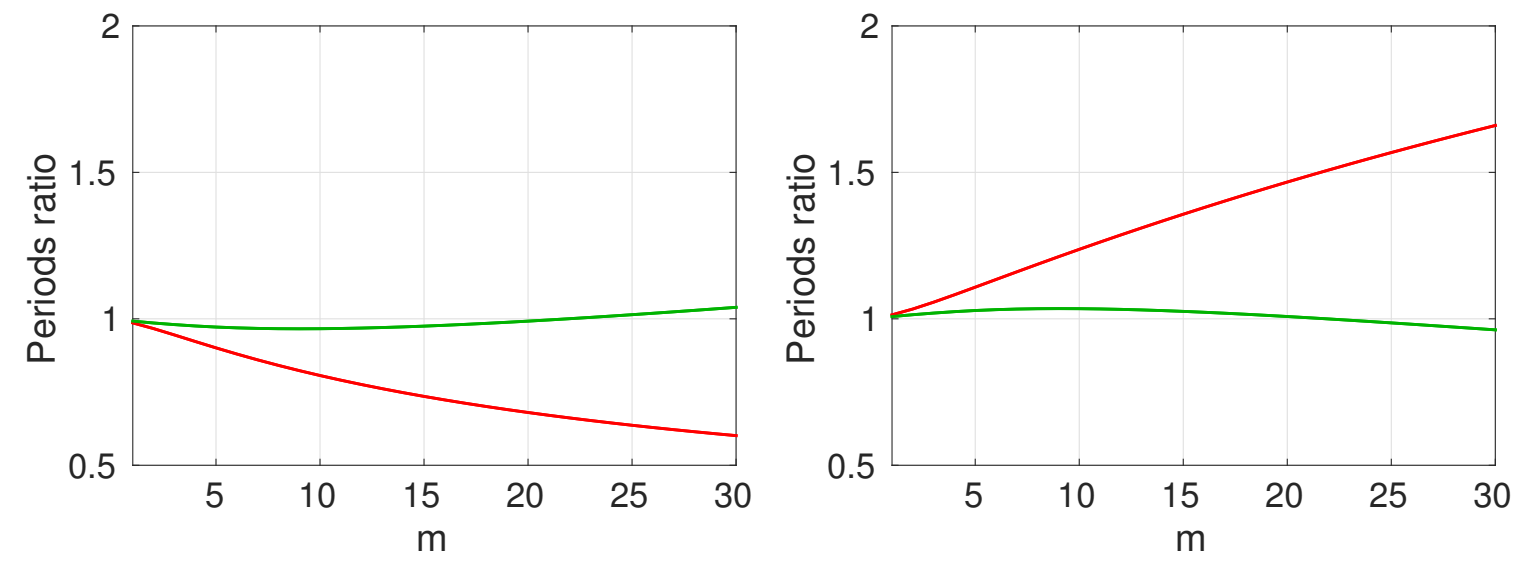

Figure 2. Hydromagnetic waves in a full sphere for Malkus' basic state. Periods of the fast (left column) and slow (right column) waves as a function of Le (top) and $m$ (middle) for $m=6$ and Le $=10^{-4}$ respectively. Black lines show the analytic results obtained by combining the equation (23) from Malkus (1967) with the expression (24) of Zhang et al. (2001) for Rossby waves. Green and red lines are the numerical results obtained with the operators $\mathcal{L}^{2}$ and $\mathcal{L}_{\zeta}^{2}$ respectively. Radial harmonics $N=1,3,6,10$ and 15 from bottom to top (fast waves) and vice-versa (slow branches). Bottom figure: ratio between estimates of $T$ from the QG reduced models and the three-dimensional primitive model for $N=3$ (green, QG model using $\mathcal{L}^{2}$; red, model using $\mathcal{L}_{\zeta}^{2}$ ) (colour online). 

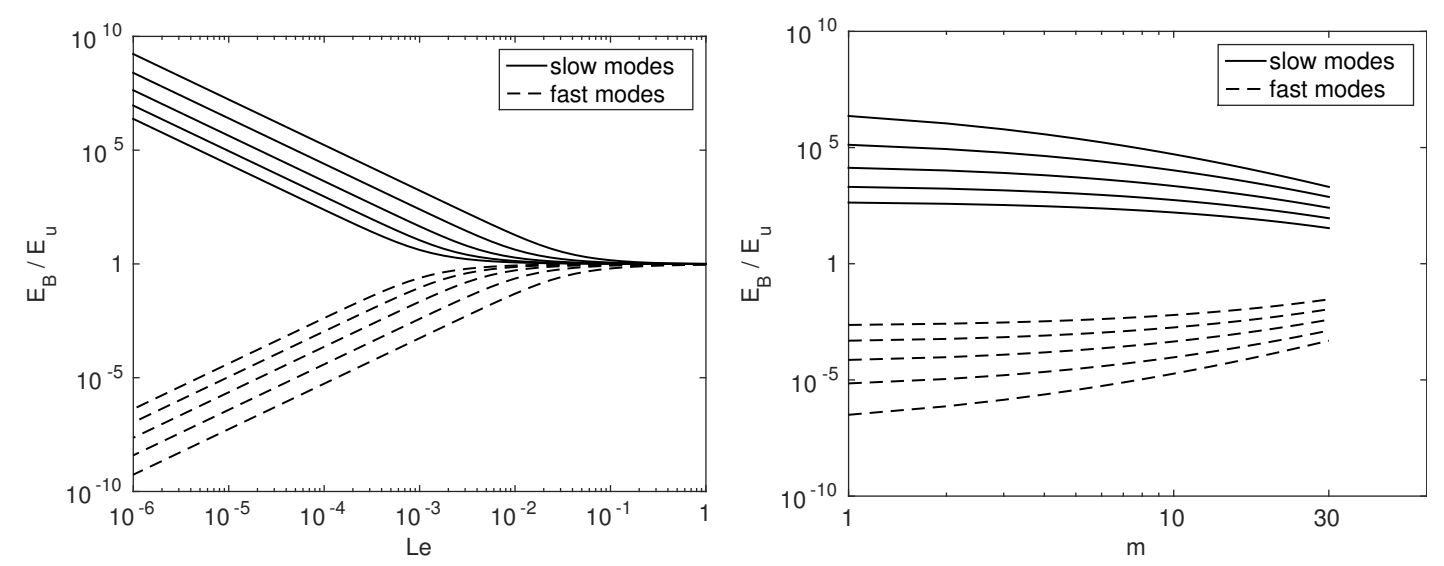

Figure 3. Malkus' basic state. Ratio of magnetic to kinetic energy as a function of Le (left) and $m$ (right) for $m=6$ and $\mathrm{Le}=10^{-4}$ respectively.

parameter $\alpha$ to zero gives us the Malkus field. We use $f(s)=-\bar{A}^{Z}(s)=-\frac{1}{3} H^{3}$ here. This choice for $f$ was made by sake of mathematical simplicity.

The section is divided into three parts. In the first, we investigate what happens when a unique harmonic component $m=m_{1}$ is added to $\bar{A}$ and, in the second, when more harmonics are included. Finally, we examine the influence of an axisymmetric $\bar{B}_{s}$.

\subsection{Torsional Alfvén waves}

Since the basic field, for $\alpha \neq 0$, has a radial component $\bar{B}_{s}$, the fluid can support torsional waves $(T W)$. Their kinetic component mainly consists in geostrophic motions. Retaining only this part of the flow, the induction equation becomes

$$
\frac{\partial \widetilde{A}}{\partial t}=-\zeta_{G} \frac{\partial \bar{A}}{\partial \phi} .
$$

By coupling the time derivative of (20) with (31), we obtain the standard torsional wave equation (Jault 2003, Roberts and Aurnou 2012)

$$
\frac{\partial^{2} \zeta_{G}}{\partial t^{2}}=\frac{1}{2 \pi s^{3} H} \frac{\partial}{\partial s}\left(s^{3} H \oint \bar{B}_{s}^{2} \mathrm{~d} \phi \frac{\partial \zeta_{G}}{\partial s}\right) .
$$

We therefore expect $T W$ to be present among the eigenmodes of our system. Their propagation in the $\pm s$ direction depends on $\bar{B}_{s}$ and slows down where $\bar{B}_{s}$ is small. If $\bar{B}_{s}$ vanishes for some $s$, incoming waves will accumulate kinetic energy there. In a modal study, this results in spatial oscillations whose length scale is equal to the resolution of the numerical scheme. To avoid this artefact, we choose a main field with no zeroes for $\bar{B}_{s}$ inside the domain. The $\bar{B}_{s}$ component does not vanish at the inner boundary $s=s_{i}$. However, the relation (17) between $\overline{\boldsymbol{B}}$ and $\bar{A}$ implies $\bar{B}_{r}=0$ on the container surface. Thus, we necessarily have $\bar{B}_{s}=\bar{B}_{r}=0$ on the equator. Therefore, we slightly shift the core boundary to the interior at $s_{o}=0.95$ - following a procedure analogous to Yano et al. (2005) - and we transfer the boundary conditions on $\psi$ and $\widetilde{A}$ initially at $s=1$ to the new boundary $s=s_{o}$ (see Figure 1 ).

Adding a unique harmonic component $m=m_{1}$ to $\bar{A}$, all modes with $m \neq m_{1}$ now involve components of wavenumber $m \pm k m_{1}$, with $k$ an integer, due to interactions with the $m=m_{1}$ component of $\overline{\boldsymbol{B}}$. Relative amplitude of components $m$ and $m \pm m_{1}$ depends on $\alpha$. At small $\alpha$ (compared to 1 ), components $m \pm m_{1}$ are negligible compared to component $m$. Modes with $m \neq m_{1}$ remain then very similar to the modes described, for $\alpha=0$, in the previous section. 
Table 1. Periods of the torsional waves.

\begin{tabular}{lcccc}
\hline & $\mathrm{N}=1$ & $\mathrm{~N}=2$ & $\mathrm{~N}=3$ & $\mathrm{~N}=4$ \\
\hline$T_{1 D}{ }^{(*)}$ & 1003.90 & 483.14 & 317.15 & 236.24 \\
$T_{Q G}{ }^{(*)}$ & 1003.87 & 483.13 & 317.13 & 236.21 \\
\hline${ }^{(*)} T_{1 D}$ obtained from the standard & equation \\
$(32),{ }^{(* *)} T_{Q G}$ period on the $T W$ branch.
\end{tabular}

At large Le, no significant change either appears in shape and period of the $M C$ waves of harmonic $m=m_{1}$. However, these $m_{1}$-dominated slow $M C$ waves are transformed into torsional waves as Le is decreased. This is illustrated in figure 4 using $m_{1}=2$. The value of Le at which this metamorphosis occurs depends on $\bar{B}_{s}$, as measured by $\alpha$. There, the $T W$ period $\left(\propto \alpha^{-1}\right)$ equals the $M C$ period $\left(\propto \mathrm{Le}^{-1}\right)$. As a result of their transformation into $T W$, the slow modes of wavenumber $m_{1}$ are strongly modified. Their period no longer depends on Le and the flow is now dominated by its zonal component, which has amplitude $\mathrm{Le}^{-1}$ higher than the component of wavenumber $m_{1}$. Finally, we have calculated independently the solutions of the standard $T W$ equation (32) (with the boundary condition $\zeta_{G}=0$ at $s=s_{i}$ and $s=s_{o}$ ). Both the periods (see Table 1) and the wave forms (see Figure 5) are in good agreement. Finally, inertial waves are unaffected by the presence of the non-axisymmetric field.

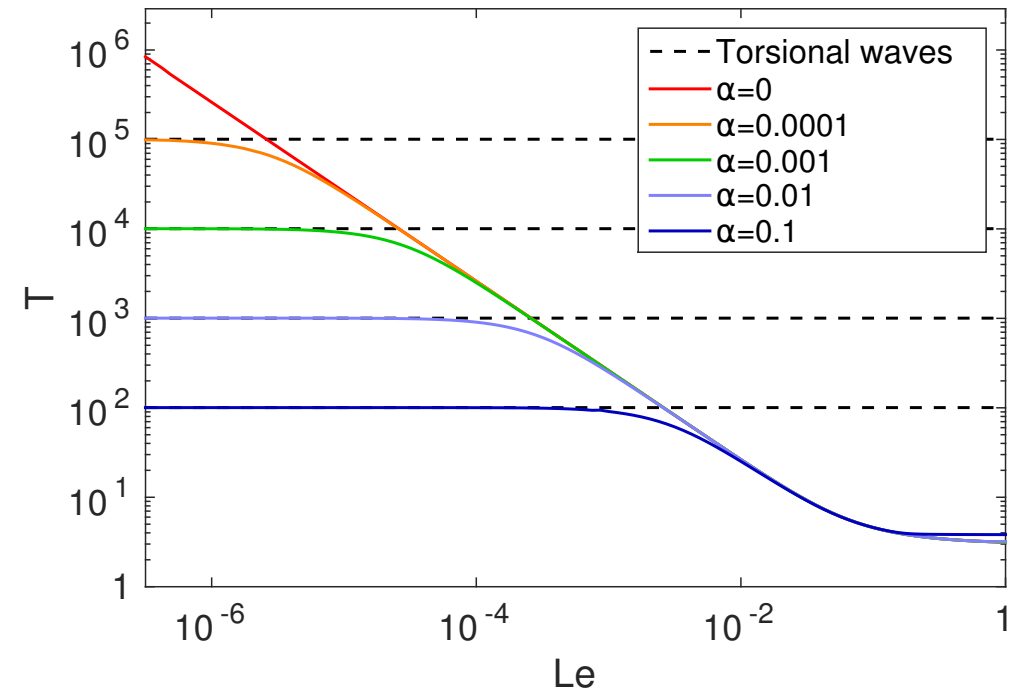

Figure 4. Periods of the waves on the slow branch as a function of Le, for $\bar{A}$ containing a unique harmonic $m_{1}=2$ in addition to $\bar{A}^{Z}$. Only the periods of the $m_{1}$ dominated waves with the largest radial scale are reported. The periods of torsional Alfvén waves found using equation (32) for the different values of $\alpha$ investigated here are displayed as dashed lines (colour online).

\subsection{A constraint on $M C$ waves}

Let us now consider $\bar{A}$ with a second harmonic $m_{2}$. At high Le, the behavior of normal modes of wavenumbers $m_{1}$ and $m_{2}$ is unchanged. The inertial and $M C$ branches separate again for Le $<1$ (see Figure 6 obtained for $m_{1}=2$ and $m_{2}=5$ ). For small Le, $T W$ are still present, with the same characteristics as before except that their non-zonal part now contains the two harmonics $m_{1}$ and $m_{2}$. The $T W$ branch is, as expected, characterized by 


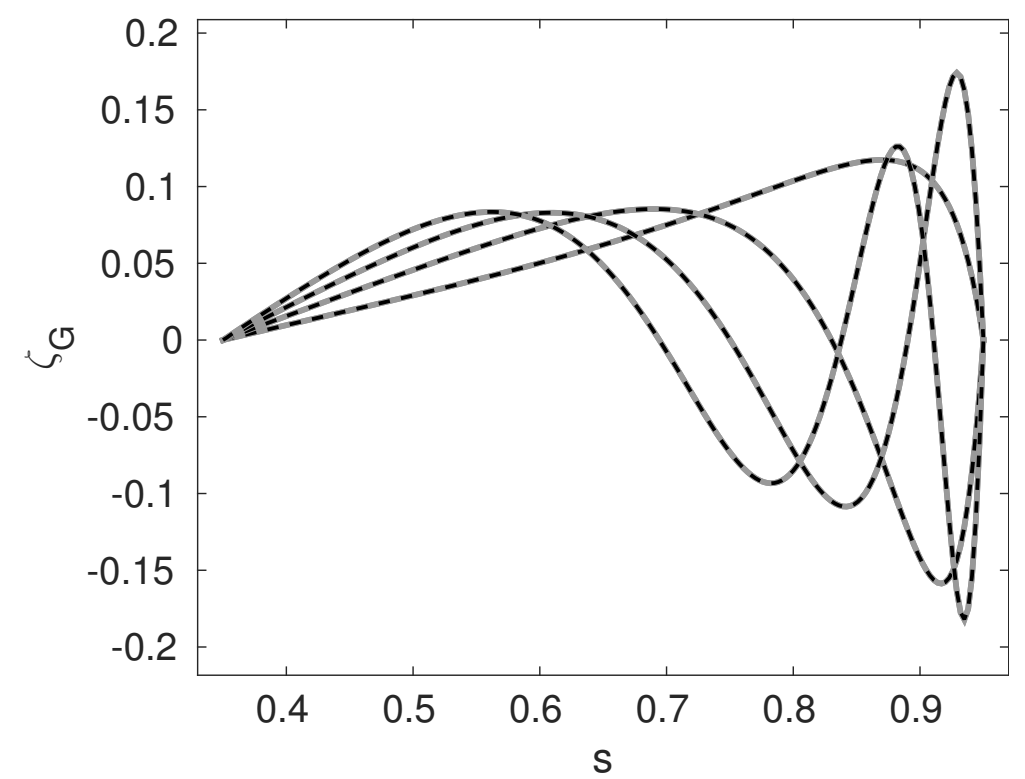

Figure 5. A comparison between the solutions of the standard equation (32) (light gray line) and the eigenmodes of the coupled QG equations (19d), (20) and (18e) (dashed black line). The basic field contains only the harmonics $m_{0}=0$ and $m_{1}=2$. The four slowest torsional waves are displayed for $\mathrm{Le}=10^{-6}, \alpha=0.01$. The two solutions are similarly normalized.

energy equipartition (see figure 7). In addition, we recover slow $M C$ waves, co-existing with $T W$. They include components of harmonic numbers $0, m_{1}$ and $m_{2}$. These new modes present the characteristics of $M C$ waves with predominant magnetic energy and periods scaling as $\mathrm{Le}^{-1}$. However, while the $M C$ waves supported by an axisymmetric basic field consist of a single harmonic $m$, these new waves combine $m_{1}$ and $m_{2}$ harmonics. In addition to the main retrograde component, there is now a small (for $\alpha \ll 1$ ) prograde constituent. Figure 8 displays the retrograde component. It shows the transformation of a purely $m_{1}$ mode into a $\left(m_{1}, m_{2}\right)$ mode. As Le is lowered, the $m_{2}$ component of both $\psi$ and $\widetilde{A}$ grows to the detriment of the $m_{1}$ component until an asymptotic state, which consists of the two harmonics $m_{1}$ and $m_{2}$ in a proportion independent of Le (for Le $\ll 1$ ), is reached. Then, the ratio of the two harmonics in $\widetilde{A}$ is inversely proportional to the ratio of the two harmonics in $\bar{A}$. Finally, at small $\alpha$, we observe that the zonal component of $\psi$ (not shown in figure 8 ) dominates; the amplitude ratio between zonal and non-zonal components scales approximately as $\alpha^{-1}$. Thus, the limit $\alpha \rightarrow 0$ is singular. At the same time, the zonal contribution to $\widetilde{A}$ remains negligible. For this reason, the ratio of magnetic to kinetic energy is particularly affected at small $\alpha$ (figure 7).

Because the $M C$ waves have low frequencies, the $\partial \boldsymbol{u} / \partial t$ term is negligible in the force balance and the waves satisfy Taylor's constraint. Taylor (1963) showed that in a rotating fluid enclosed in a rigid boundary, a solution of the magnetostrophic equations can exist only if the Lorentz force integrated over geostrophic cylinders vanishes, see equation (1b). In our model, Taylor's constraint can be written as

$$
\forall s, \quad \oint\left(\frac{\partial \bar{A}}{\partial s} \frac{\partial \widetilde{A}}{\partial \phi}+\frac{\partial \widetilde{A}}{\partial s} \frac{\partial \bar{A}}{\partial \phi}\right) \mathrm{d} \phi=0
$$

We quantify the Taylor's contraint on $M C$ waves by estimating the 'Taylorisation' $\mathcal{T}$ (Walker 


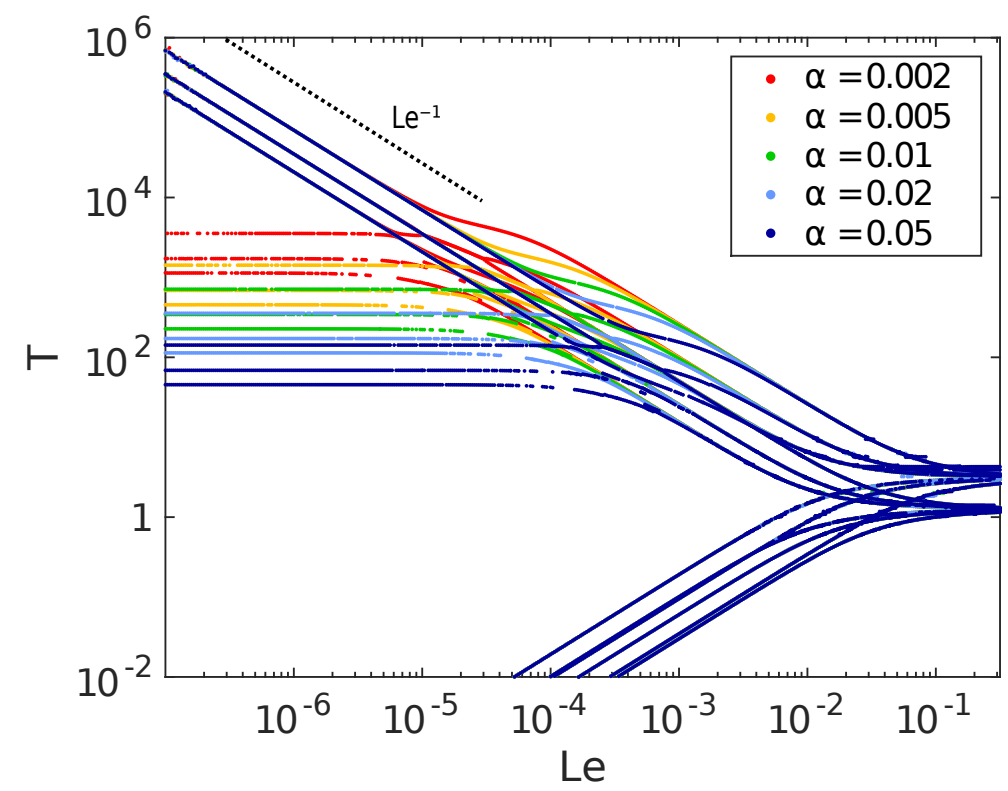

Figure 6. Impact of $\alpha$ on the separation between the $M C$ and $T W$ branches, in a case where $\bar{A}$ contains contributions of wavenumbers $m_{0}=0, m_{1}=2$ and $m_{2}=5$. Modes combining $m_{1}$ and $m_{2}$ components. Periods as a function of the Lehnert number Le. In the Le $\rightarrow 0$ limit, the periods of $M C$ waves are independent of $\alpha . T W$ periods vary as $\alpha^{-1}$ (colour online).

and Barenghi 1998) as a function of Le:

$$
\mathcal{T}=\frac{\int_{s_{i}}^{s_{o}} \oint\left(\frac{\partial \bar{A}}{\partial s} \frac{\partial \widetilde{A}}{\partial \phi}+\frac{\partial \widetilde{A}}{\partial s} \frac{\partial \bar{A}}{\partial \phi}\right) \mathrm{d} \phi \mathrm{d} s}{\int_{s_{i}}^{s_{o}} \oint\left|\frac{\partial \bar{A}}{\partial s} \frac{\partial \widetilde{A}}{\partial \phi}+\frac{\partial \widetilde{A}}{\partial s} \frac{\partial \bar{A}}{\partial \phi}\right| \mathrm{d} \phi \mathrm{d} s} .
$$

We find $\mathcal{T}$ to be of the order $10^{-3}$ at $\mathrm{Le}=10^{-5}$ and to vary as $\mathrm{Le}^{2}$ (see figure 9). Taylor's constraint is thus well satisfied by the $M C$ modes that combine components with different wavenumbers.

In accordance with another idea of Taylor (1963), we substitute $\partial \widetilde{A} / \partial t$ from the induction equation (18e) in the time derivative of (33) and obtain a linear relationship between $\zeta_{G}$ and the non-zonal part of the stream function $\psi^{N Z}$ :

$$
\frac{\partial \zeta_{G}}{\partial s}=\frac{\oint\left[\frac{\partial}{\partial s}\left(\frac{1}{H} \mathcal{J}\left(\psi^{N Z}, \bar{A}\right)\right) \frac{\partial \bar{A}}{\partial \phi}+\frac{\partial}{\partial \phi}\left(\frac{1}{H} \mathcal{J}\left(\psi^{N Z}, \bar{A}\right)\right) \frac{\partial \bar{A}}{\partial s}\right] \mathrm{d} \phi}{\oint\left(\frac{\partial \bar{A}}{\partial \phi}\right)^{2} \mathrm{~d} \phi} .
$$

Analysis of orders of magnitude in (35) indicates that $\left(\psi_{G} / \psi^{N Z}\right)$ is expected to scale as $\frac{1}{\alpha}$ when $\alpha \ll 1$. Applying equation (35) to the non-zonal stream function $\psi^{N Z}$ for $M C$ eigenmodes, we get an indirect estimate of the geostrophic shear for each of these modes that can be compared to its actual value. The remarkable agreement that we find (see figure 10) gives yet another proof that the magnetic field carried by $M C$ waves obeys Taylor's constraint (33). 

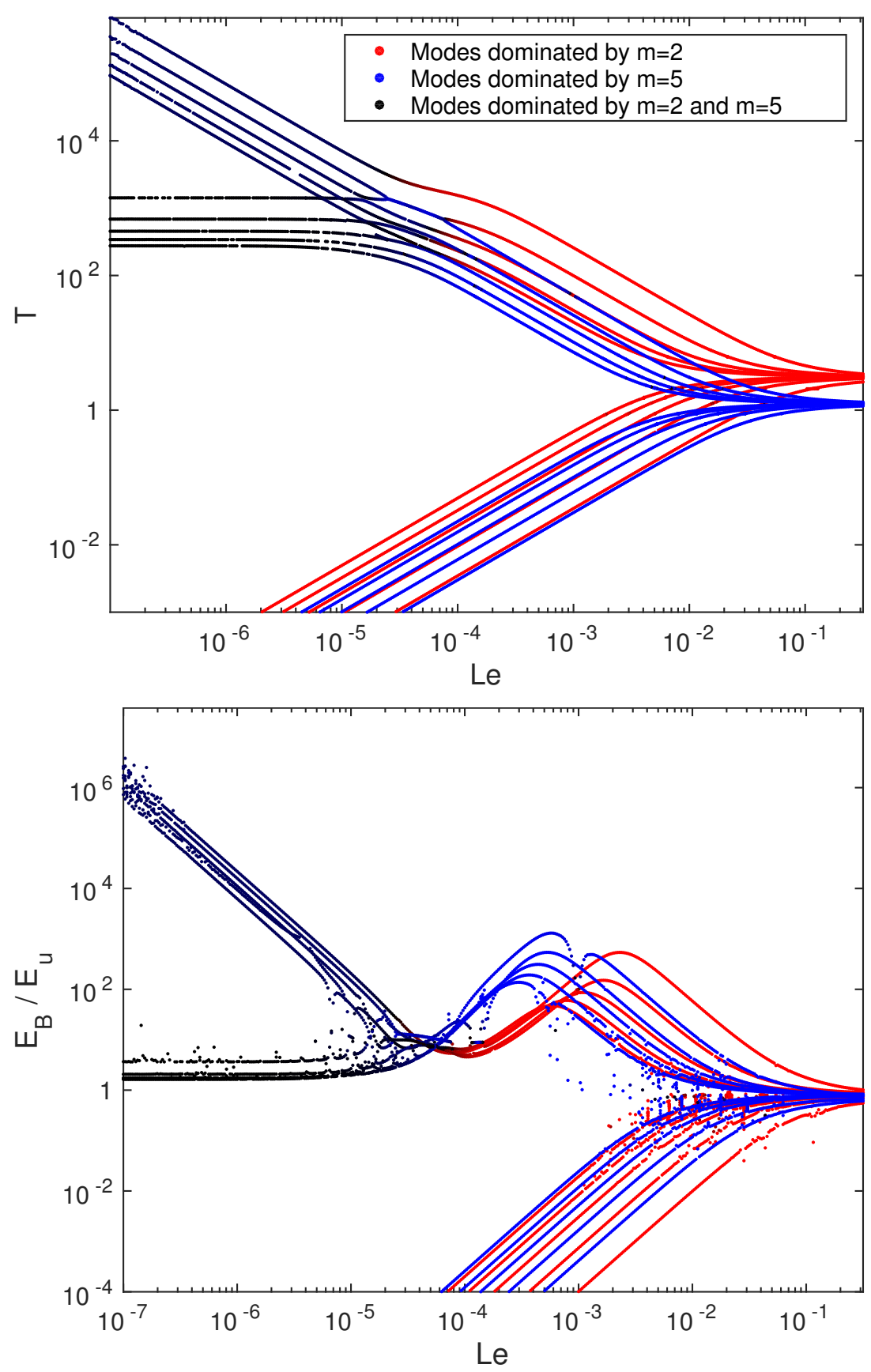

Figure 7. Separation between the $M C$ and $T W$ branches when $\bar{A}$ contains the azimuthal wavenumbers $m_{0}=0, m_{1}=2$ and $m_{2}=5$, with $\alpha=5 \cdot 10^{-3}$. Shown are eigenmodes whose non-axisymmetric part is dominated by wavenumbers $m_{1}$ and $m_{2}$ (restricted to the modes of lowest radial complexity). Top panel: periods of the different modes. Bottom panel: Ratio of magnetic energy to kinetic energy. The $M C$ branch is characterized by increasingly dominant magnetic energy as Le is lowered. The inertial branch is characterized by dominant kinetic energy, and there is approximately energy equipartition on the $T W$ branch. If two modes have the same eigenvalue, the algorithm finds a single eigenmode which is a linear combination of both modes. Due to numerical error, this also happens when eigenfrequencies are close to one another (e.g. on top panel, every time a blue line crosses a red line), and some physically unrelated modes are then calculated as one. This results in dispersion in the energy ratio (bottom figure), and some modes being ignored by the plotting algorithm (missing points on the top figure) (colour online). 

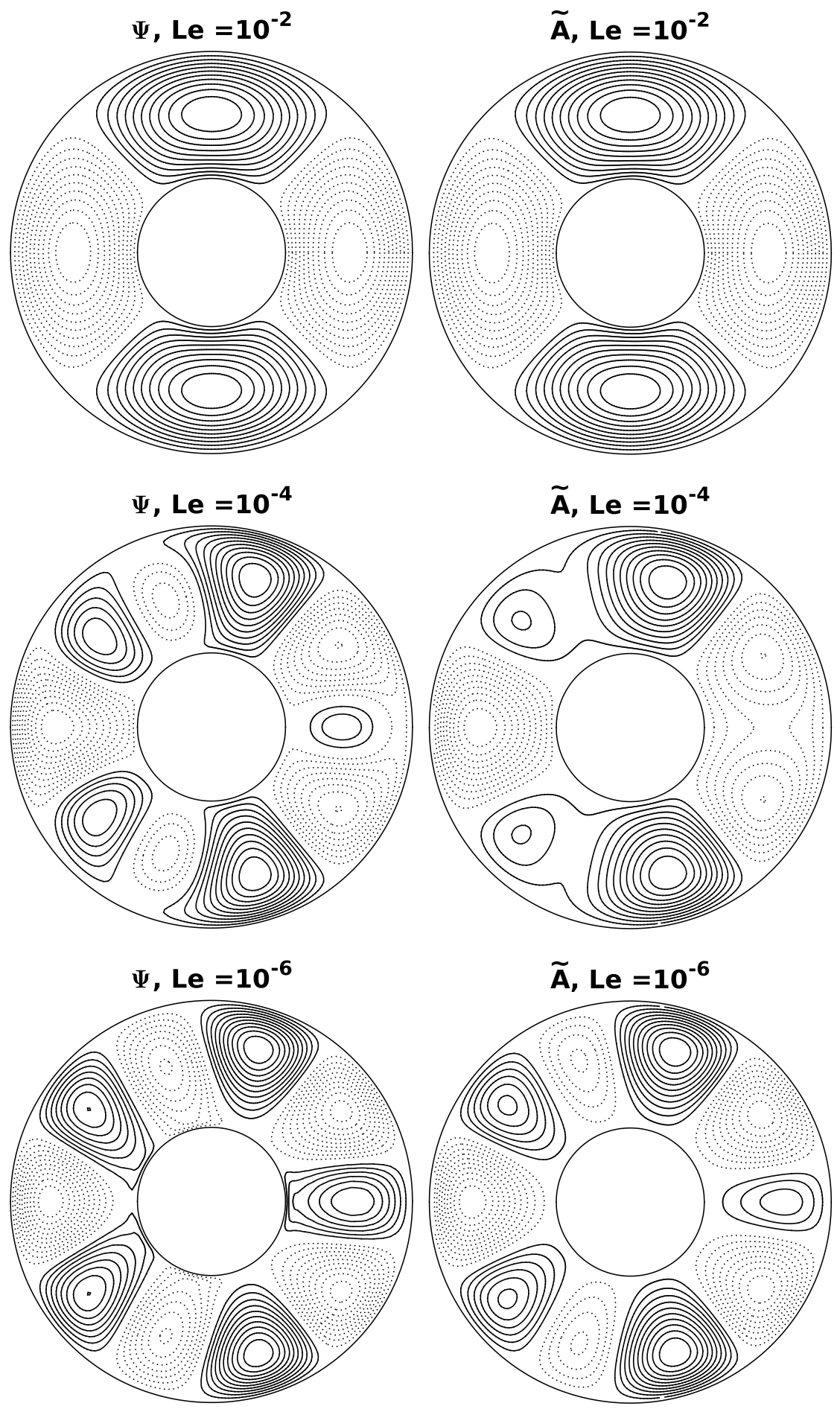

Figure 8. Examples of slow $M C$ waves when $\bar{A}$ contains the harmonics $m_{0}=0$, $m_{1}=2$ and $m_{2}=5$. Transformation, as Le is decreased, of the non-zonal and retrograde component of $\psi$ (left) and $\widetilde{A}$ (right) for the mode originally dominated by $m=2$ and of simplest radial complexity . This mode corresponds to the uppermost curve in figure 7 . At large Le, it is similar to the mode $(m=2, N=1)$ found with purely axisymmetric $\bar{A}$. As Le decreases, the $m=5$ component grows. 


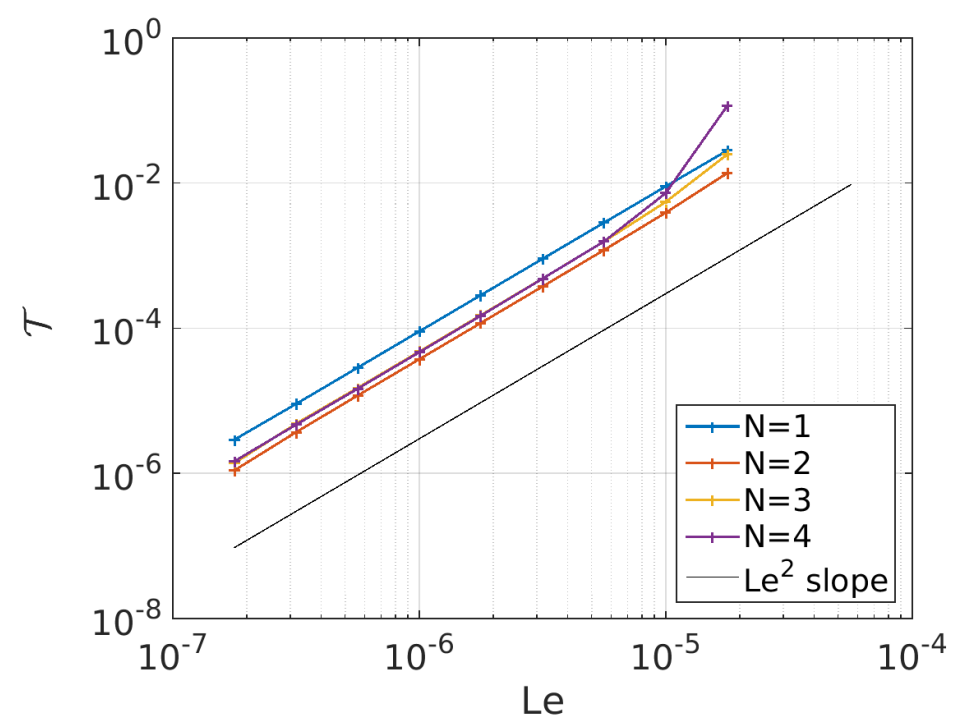

Figure 9. 'Taylorization' of the $M C$ modes dominated by harmonics $m_{1}$ and $m_{2}$. Simplest radial scale modes are displayed for $\alpha=10^{-2}$. $\mathcal{T}$ evolves as $\mathrm{Le}^{2}$ for small Le. This ceases to be true above Le $=10^{-5}$, as $M C$ and $T W$ branches merge (colour online).

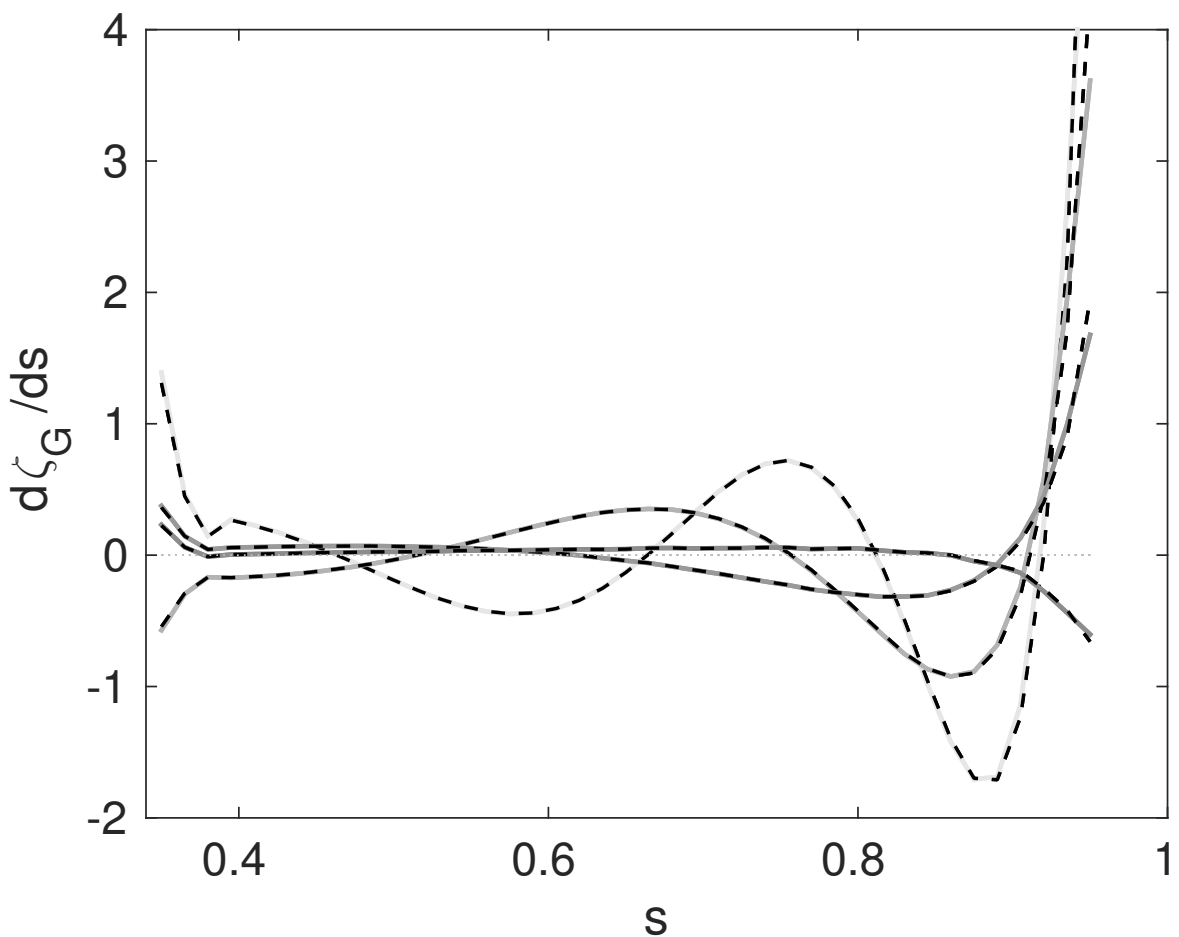

Figure 10. A comparison between two estimations of the geostrophic shear for $m_{1}=2$, $m_{2}=5 M C$ waves. The shear $\frac{\partial \zeta_{G}}{\partial s}$ is either derived from $\psi^{N Z}$ assuming Taylor's constraint by (35) (dashed black) or obtained directly as the zonal velocity ingredient of the wave (light grey). The first four radial harmonics are displayed. $\alpha=10^{-2}, \mathrm{Le}=10^{-6}$. 


\subsection{Adding an axisymmetric poloidal component to the imposed magnetic field}

Thus far, we have limited the space of solutions (and of basic fields) to magnetic fields defined from a $z$-invariant scalar potential $A(s, \phi)$. This model lacks important ingredients. It does not reproduce the creation of toroidal zonal field from the shear of the poloidal magnetic field by the zonal velocity. As a result, the dominance of the zonal velocity in the kinetic energy budget for $M C$ waves at small $\alpha$ is not reflected in the apportion of magnetic energy. Also, the behavior of the ratio $\left(\psi_{G} / \psi^{N Z}\right)$ is singular at $\alpha=0$, since the $M C$ waves have no $m=0$ ingredients in the case of the Malkus field $(\alpha=0)$. In order to remediate this singularity, we allow for an axial dipolar field $\overline{\boldsymbol{B}}_{d}$ in the basic field,

$$
\overline{\boldsymbol{B}}=\frac{1}{H} \boldsymbol{\nabla} \times\left(\bar{A} \mathbf{1}_{\boldsymbol{z}}\right)+z \frac{\beta}{s H} \frac{\partial \bar{A}}{\partial \phi} \mathbf{1}_{\boldsymbol{z}}+\delta \overline{\boldsymbol{B}}_{d}(s, z),
$$

where $\bar{A}$ is described in (30), and the small parameter $\delta$ serves a purpose similar to $\alpha$.

The expression of $\overline{\boldsymbol{B}}_{d}$ in cylindrical coordinates is

$$
\overline{\boldsymbol{B}}_{d}=\left(\frac{-3 s z}{\left(s^{2}+z^{2}\right)^{\frac{5}{2}}}, 0, \frac{s^{2}-2 z^{2}}{\left(s^{2}+z^{2}\right)^{\frac{5}{2}}}\right) .
$$

Note that we can still ensure $\overline{\boldsymbol{B}}_{r}(1)=0$ by adding an uniform field along $\mathbf{1}_{\boldsymbol{z}}$. We take $\overline{\boldsymbol{B}}_{d}$ into account only in the evolution equation for the geostrophic velocity (11a), for which it yields an additional term $\frac{1}{2 s^{3} H} \frac{\partial}{\partial s}\left(s^{2} q_{s \phi}\right)$ to the right hand side (Jault 2003, Roberts and Aurnou 2012). We modify equation (20) accordingly.

$$
\frac{\partial \zeta_{G}}{\partial t}=-\frac{1}{2 \pi s^{3} H} \oint\left(\frac{\partial}{\partial s}\left(\frac{s}{H} \frac{\partial \bar{A}}{\partial s}\right) \frac{\partial \widetilde{A}}{\partial \phi}+\frac{\partial \bar{A}}{\partial \phi} \frac{\partial}{\partial s}\left(\frac{s}{H} \frac{\partial \widetilde{A}}{\partial s}\right)\right) \mathrm{d} \phi+\frac{1}{2 s^{3} H} \frac{\partial}{\partial s}\left(s^{2} q_{s \phi}\right) .
$$

The quantity $q_{s \phi}$,

$$
q_{s \phi}(s)=\left\{\bar{B}_{d, s} \widetilde{B}_{\phi}(s)\right\},
$$

arises from an induced field that is antisymmetric with respect to the equator and cannot be represented via the $z$-invariant scalar potential $\widetilde{A}$. Finally, a new equation is needed to account for the time evolution of $q_{s \phi}$. From the induction equation, we deduce :

$$
\frac{\partial}{\partial t} q_{s \phi}=s\left\{\bar{B}_{d, s}^{2}\right\} \frac{\partial \zeta_{G}}{\partial s} .
$$

In the absence of a non-axisymmetric part in $\overline{\boldsymbol{B}}(\alpha=0)$, purely zonal $T W$ carried by the dipole coexists with the purely non-axisymmetric modes described in $\S 3.2$ carried by Malkus' field. Those two sets of solutions are contained in two orthogonal spaces (zonal and non-zonal), and have therefore no influence on each other.

Now, we return to the case when there are two harmonic components $m_{1}$ and $m_{2}$ in $\bar{A}$ (of small amplitude $\alpha$ ). When the dipole field is small in comparison with the non-axisymmetric component of $\overline{\boldsymbol{B}}(\delta \ll \alpha)$, the solutions remain similar to the ones discussed in the previous section, with the additional presence of low frequency torsional waves (of period independent of Le) supported by the dipole field (top row of figure 11). The latter waves morph into $M C$ waves at the value of Le for which part of the $M C$ waves that owe their existence to the non-dipole field are changed into torsional waves. Investigating the other extreme, when the dipole gets strong compared to the non-axisymmetric part of $\bar{B}(\alpha \ll \delta)$, we find that $M C$ waves evolve toward the modes found with the purely axisymmetric Malkus' field (bottom row of figure 11). Thus, decreasing $\alpha$ while keeping $\delta$ constant removes the singularity in the 
behavior of $\left(\psi_{G} / \psi^{N Z}\right)$. Also, the zonal velocity, which is intense on the $M C$ branch for $\delta \ll \alpha$ and small Le, vanishes for $\alpha \ll \delta$. As a result, there is not any more the kink in the energy ratio that arises when $m_{1}$ and $m_{2} M C$ waves combine into mixed torsional and $M C$ waves. In the intermediate case $(\delta \approx \alpha)$, the periods of the torsional waves supported by the dipole are about the same as the periods of the torsional waves supported by the non-axisymmetric part of $\bar{B}_{s}$.

Finally, let us discuss the case when there is only one harmonic component $m_{1}$ in $\bar{A}$. In the presence of a significant dipole field $(\alpha \ll \delta), M C$ waves resuscitate (Figure 12 right). Then, we recover all the waves supported by the Malkus' field. In the opposite situation, very slow $M C$ waves with $B_{s}$ mainly of wavenumber $m=0$ but with a small $m=m_{1}$ component arise together with torsional waves whose magnetic part is mainly of wavenumber $m_{1}$ (Figure 12 left).

\section{Concluding remarks}

We have presented the first description of $M C$ waves in the presence of a non-axisymmetric magnetic field. In this configuration, torsional Alfvén modes and $M C$ modes arise simultaneously: the torsional waves are supported by the interaction between the non-axisymmetric magnetic part of the wave and the basic magnetic field. This illustrates a possible mechanism for the excitation of these waves in the Earth's core (Gillet et al. 2015, Teed et al. 2015).

The $M C$ branches are characterized at small Le. Then, they span an hyperplane of the space of solutions defined by Taylor's constraint. This constraint can indeed be interpreted as a projection that depends on the ambient magnetic field $\overline{\boldsymbol{B}}$ (see the related approach by Livermore et al. (2011)). When the $s$-component of $\overline{\boldsymbol{B}}$ contains only one harmonic number $m_{1}$ as in $\S 4.1$, the hyperplane simply consists of the waves with $m \neq m_{1}$, which are thus unaffected by the constraint, and there are no $m=m_{1} M C$ waves. Section $\S 4.3$ shows that this description holds also in the case $m_{1}=0$. When the $s$-component of $\overline{\boldsymbol{B}}$ contains two harmonic numbers $m_{1}$ and $m_{2}$ as in $\S 4.2$, description of the hyperplane is less trivial. It includes all waves with $m \neq m_{1}$ and $m \neq m_{2}$ components. There are also $M C$ waves with $m=m_{1}$ and $m=m_{2}$ components. Both the $T W$ and the $M C$ branches combine indeed $m_{1}$ and $m_{2}$ parts in proportions set by the Taylor's constraint. This result can be generalized to imposed fields with three or more wavenumbers.

We have used a weak formulation to derive a novel $Q G$ model in a deep spherical shell with large height variations. The model reproduces the nearly geostrophic three-dimensional inertial waves and, in the case of the Malkus' field, the $M C$ waves as well. We have shown that it can be generalized, without further assumptions, to include non-linear and dissipative terms in the induction and momentum equations. These results are encouraging in the perspective of describing the time evolution of the flow within the Earth's core. The class of flows that enters the reduced model has indeed been chosen so that the interior flows can match at the core-mantle boundary core surface flows that participate in the geomagnetic secular variation (e.g. Gillet et al. 2015). Furthermore, the size of the parameter space is limited as the threedimensional velocity vector is represented through a single two-dimensional scalar. Lastly, the derivation of the model using a variational approach makes it well suited for an implementation into data assimilation algorithms (Fournier et al. 2010).

\section{Acknowledgements}

We thank David Cébron and Nathanaël Schaeffer, who have closely followed our study. We are grateful also to Mathieu Dumberry who made numerous remarks prior to the submission 

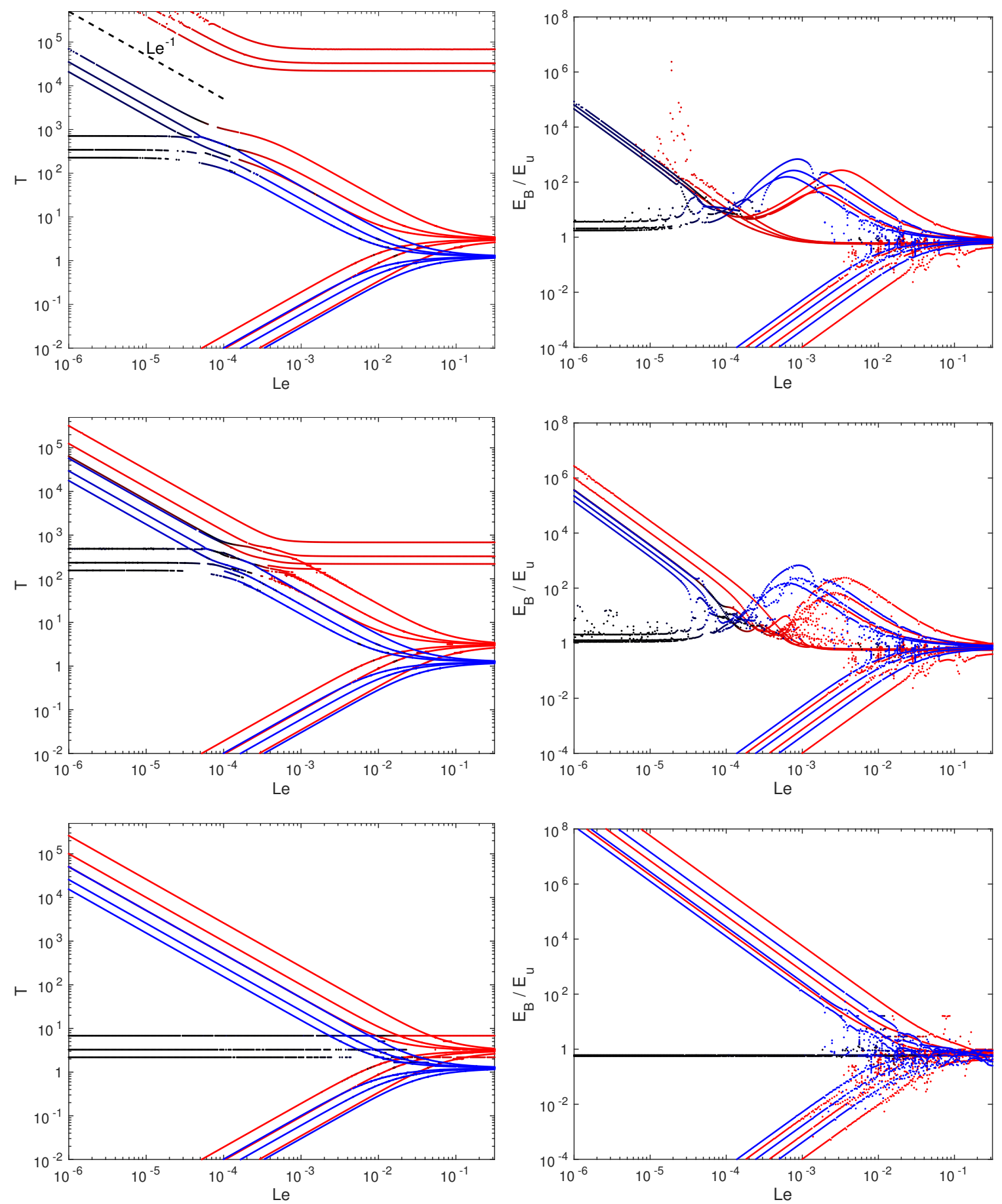

Figure 11. Periods (left) and energy ratio (right) of $M C$ waves in the presence of an axial dipole, for different values of $\delta$. From top to bottom, $\delta=10^{-5}, 10^{-3}, 10^{-1} . \alpha=10^{-2}$. Modes where the non-axisymmetric induced field is dominated by azimuthal wavenumber $m_{1}=2\left(m_{2}=5\right)$ are shown in red (blue). Modes were both $m_{1}$ and $m_{2}$ are dominant are show in black (colour online).

of the article. Discussions with Elisabeth Canet have been much helpful. We thank Phil Livermore and an anonymous referee for their precise remarks that helped improve the clarity of the manuscript. This work was partially supported by the French Centre National d'Études Spatiales (CNES) for the study of Earth's core dynamics in the context of the Swarm mission of ESA and by the French Agence Nationale de la Recherche under the grant ANR-11-BS56- 

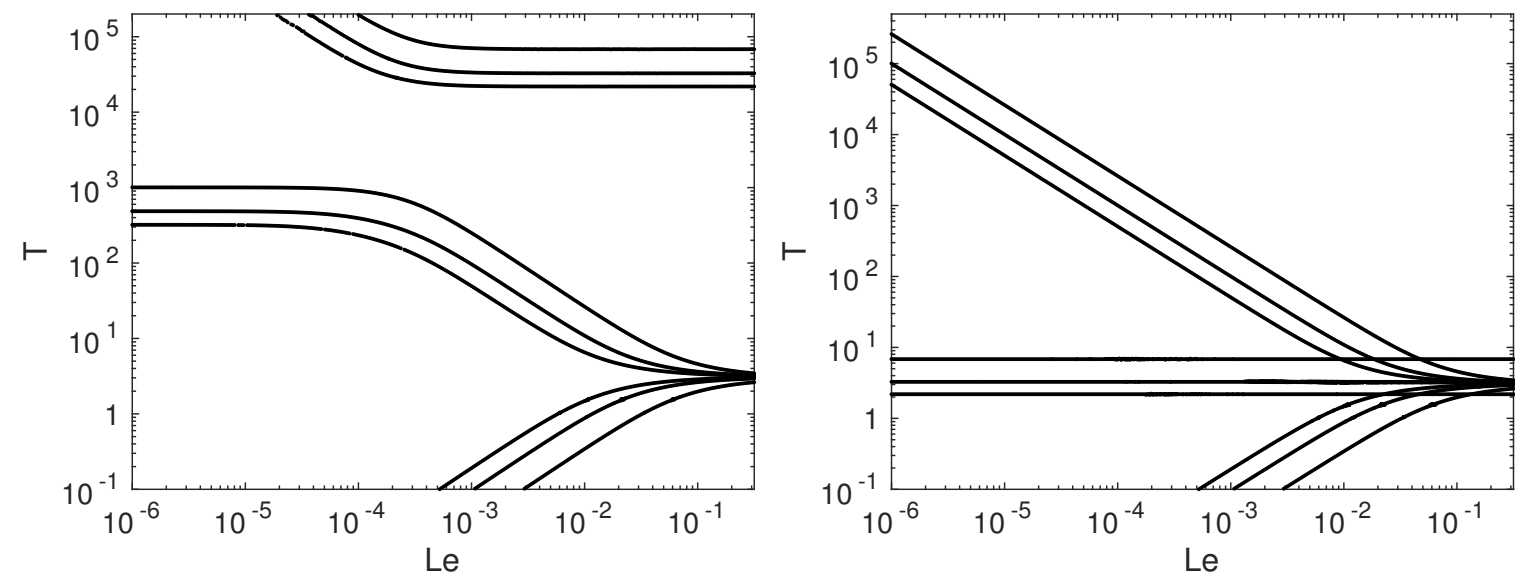

Figure 12. Periods of $M C$ waves in the presence of an axial dipole, for $\delta=10^{-5}$ (left) and $\delta=10^{-1}$ (right), with the non-axisymmetric induced field dominated by wavenumber $m_{1}=2 . \alpha=10^{-2}$.

011. ISTerre is part of Labex OSUG@2020 (ANR10 LABX56).

\section{References}

Braginsky, S.I., Torsional magnetohydrodynamic vibrations in the Earth's core and variations in day length. Geomag. Aeron., 1970, 10, 1-8.

Braginsky, S.I., Nearly axially symmetric model of the hydromagnetic dynamo of the Earth. Geomag. Aeron., 1978, 18, 225-231.

Calkins, M.A., Aurnou, J.M., Eldredge, J.D. and Julien, K., The influence of fluid properties on the morphology of core turbulence and the geomagnetic field. Earth Planet. Sci. Lett., 2012, 359-360, 55-60.

Canet, E., Finlay, C.C. and Fournier, A., Hydromagnetic modes in rapidly rotating planetary cores. Phys. Earth planet. inter., 2014, 229, 1-15.

Christensen, U., Olson, P. and Glatzmaier, G.A., Numerical modelling of the geodynamo: a systematic parameter study. Geophys. J. Int., 1999, 138, 393-409.

Finlay, C.C., Magnetohydrodynamic waves. In Encyclopedia of geomagnetism and paleomagnetism, edited by D. Gubbins and E. Herrero-Bervera, pp. 632-639, 2007 (Springer: Netherlands).

Fournier, A., Hulot, G., Jault, D., Kuang, W., Tangborn, A., Gillet, N., Canet, E., Aubert, J. and Lhuillier, F., An Introduction to Data Assimilation and Predictability in Geomagnetism. Space Science Reviews, 2010, 155, 247-291.

Gans, R.F., On hydromagnetic oscillations in a rotating cavity. J. Fluid Mech., 1971, 50, 449-467.

Gillet, N., Jault, D. and Finlay, C.C., Planetary gyre, time-dependent eddies, torsional waves and equatorial jets at the Earth's core surface. J. Geophys. Res. - Solid Earth, 2015, 120, 3991-4013.

Gillet, N. and Jones, C.A., The quasi-geostrophic model for rapidly rotating spherical convection outside the tangent cylinder. J. Fluid Mech., 2006, 554, 343-369.

Gillet, N., Jault, D., Canet, E. and Fournier, A., Fast torsional waves and strong magnetic field within the Earth's core. Nature, 2010, 465, 74-77.

Gillet, N., Schaeffer, N. and Jault, D., Rationale and geophysical evidence for quasi-geostrophic rapid dynamics within the Earth's outer core. Phys. Earth planet. inter., 2011, 187, 380-390.

Hide, R., Free Hydromagnetic Oscillations of the Earth's Core and the Theory of the Geomagnetic Secular Variation. Phil. Trans. Roy. Soc. London A, 1966, 259, 615-647.

Jault, D., Model-Z by computation and Taylor's condition. Geophys. Astrophys. Fluid Dyn., 1995, 79, $99-124$.

Jault, D., Electromagnetic and topographic coupling, and LOD variations. In Earth's core and lower mantle, edited by C. Jones, A. Soward and K. Zhang, pp. 56-76, 2003 (Taylor \& Francis: London).

Jault, D. and Finlay, C.C., Waves in the core and mechanical core-mantle interactions. In Treatise on Geophysics, Core Dynamics, 2nd edition, edited by G. Schubert and P. Olson, Vol. 8, chap. 8.09, pp. 225-244, 2015 (Elsevier: Amsterdam).

Jault, D., Axial invariance of rapidly varying diffusionless motions in the Earth's core interior. Phys. Earth planet. inter., 2008, 166, 67-76.

Livermore, P.W., Ierley, G. and Jackson, A., The construction of exact Taylor states. I: The full sphere. Geophys. J. Int., 2009, 179, 923-928.

Livermore, P.W., Ierley, G. and Jackson, A., The evolution of a magnetic field subject to Taylor's constraint using a projection operator. Geophys. J. Int., 2011, 187, 690-704.

Malkus, W.V.R., Hydromagnetic planetary waves. J. Fluid Mech., 1967, 28, 793-802. 
Pozzo, M., Davies, C., Gubbins, D. and Alfé, D., Transport properties for liquid silicon-oxygen-iron mixtures at Earth's core conditions. Physical Review B, 2013, 87, 014110.

Roberts, P.H. and Aurnou, J.M., On the theory of core-mantle coupling. Geophys. Astrophys. Fluid Dyn., 2012, 106, $157-230$.

Schaeffer, N. and Cardin, Ph., Quasigeostrophic model of the instabilities of the Stewartson layer in flat and depth-varying containers. Physics of Fluids, 2005, 17, 104111.

Soderlund, K.M., King, E.M. and Aurnou, J.M., The influence of magnetic fields in planetary dynamo models. Earth Planet. Sci. Lett., 2012, 333-334, 9-20.

Taylor, J.B., The magneto-hydrodynamics of a rotating fluid and the earth's dynamo problem. Proc. R. Soc. Lond., A, 1963, 274, 274-283.

Teed, R.J., Jones, C.A. and Tobias, S.M., The transition to Earth-like torsional oscillations in magnetoconvection simulations. Earth Planet. Sci. Lett., 2015, 419, 22-31.

Tobias, S.M., Diamond, P.H. and Hughes, D.W., $\beta$-plane magnetohydrodynamic turbulence in the solar tachocline. Astrophys. J., 2007, 667, L113-L116.

Vallis, G.K., Atmospheric and oceanic fluid dynamics, 2006 (Cambridge University Press).

Walker, M.R. and Barenghi, C.F., Accurate numerical computations of Taylor integrals. Geophys. Astrophys. Fluid Dyn., 1998, 87, 173-191.

Wu, C.C. and Roberts, P.H., On magnetostrophic mean-field solutions of the geodynamo equations. Geophys. Astrophys. Fluid Dyn., 2015, 109, 84-110.

Yano, J.I., Talagrand, O. and Drossart, P., Deep two-dimensional turbulence: An idealized model for atmospheric jets of the giant outer planets. Geophys. Astrophys. Fluid Dyn., 2005, 99, 137-150.

Zeitlin, V., Remarks on rotating shallow-water magnetohydrodynamics. Nonlin. Processes Geophys., 2013, 20, 893-898.

Zhang, K., Earnshaw, P., Liao, X. and Busse, F.H., On inertial waves in a rotating fluid sphere. J. Fluid Mech., 2001, 437, 103-119.

Zhang, K., Liao, X. and Schubert, G., Non-Axisymmetric instabilities of a toroidal magnetic field in a rotating sphere. Astrophys. J., 2003, 585, 1124-1137.

\section{Appendix A: Reduced equations with dissipative and non-linear terms}

We can write the original primitive equations (5) and (6) in reduced form. From (16), we find that

$$
\begin{aligned}
& \boldsymbol{\nabla} \times \boldsymbol{\nabla} \times \boldsymbol{B}=\mathbf{1}_{\boldsymbol{z}} \times \boldsymbol{\nabla}\left(\mathcal{L}_{\zeta}^{2} A\right)+\boldsymbol{\nabla}\left(\frac{\beta}{s H} \frac{\partial A}{\partial \phi}\right) \\
& -z \mathbf{1}_{\boldsymbol{z}}\left(\frac{1}{s} \frac{\partial}{\partial s}\left(s \frac{\partial}{\partial s}\left(\frac{\beta}{s H} \frac{\partial A}{\partial \phi}\right)\right)+\frac{\beta}{s^{3} H} \frac{\partial^{3} A}{\partial \phi^{3}}\right),
\end{aligned}
$$

where $\mathcal{L}_{\zeta}^{2}$ is defined in (14). Restricting the derivation to the spherical case, for which $\beta=$ $-s^{2} / H$, projection onto trial functions $\boldsymbol{B}^{\prime}\left(A^{\prime}\right)$ gives

$$
\iiint \boldsymbol{B}^{\prime} \cdot \boldsymbol{\nabla} \times \boldsymbol{\nabla} \times \boldsymbol{B} \mathrm{d} \mathcal{V}=2 \iint A^{\prime} \nabla^{2}\left(\mathcal{L}^{2} A\right) \mathrm{d} \mathcal{A},
$$

where $\nabla^{2}$ denotes the Laplacian. The magnetic field derivative transforms as the acceleration in (13e). Therefore, by (18d), the induction equation (6) is reduced to

$$
\frac{\partial}{\partial t} \mathcal{L}^{2} A=\mathcal{L}^{2}\left(\frac{1}{H} \mathcal{J}(\psi, A)\right)+\operatorname{Lu}^{-1} \nabla^{2}\left(\mathcal{L}^{2} A\right) .
$$

Similarly, the Navier-Stokes equation (5) can be transformed into

$$
\frac{\partial}{\partial t} \mathcal{L}^{2} \psi-\mathcal{J}\left(\psi, \frac{1}{H} \mathcal{L}^{2} \psi\right)+\frac{2}{\operatorname{Le}} \frac{\beta}{s H} \frac{\partial \psi}{\partial \phi}=-\mathcal{J}\left(A, \frac{1}{H} \mathcal{L}^{2} A\right)+\mathrm{P}_{\mathrm{m}} \mathrm{Lu}^{-1} \nabla^{2}\left(\mathcal{L}^{2} \psi\right)
$$

since the non-linear term $\boldsymbol{u} \cdot \boldsymbol{\nabla} \boldsymbol{u}$ can be treated as the magnetic force. For the sake of completeness, we add the equation for the geostrophic velocity

$$
\frac{\partial u_{G}}{\partial t}=-\frac{1}{2 \pi s^{2} H} \oint\left(\frac{\partial}{\partial s}\left(\frac{s}{H} \frac{\partial A}{\partial s}\right) \frac{\partial A}{\partial \phi}\right) \mathrm{d} \phi+\mathrm{P}_{\mathrm{m}} \mathrm{Lu}^{-1} \nabla^{2} u_{G} .
$$


Geophysical and Astrophysical Fluid Dynamics $\quad$ article

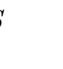

